\title{
A Simplified Output Regulator for a Class of Takagi-Sugeno Fuzzy Models
}

\author{
Tonatiuh Hernández-Cortés, Jesús A. Meda Campaña, \\ Luis A. Páramo Carranza, and Julio C. Gómez Mancilla
}

Instituto Politécnico Nacional, SEPI-ESIME Zacatenco, Avenue IPN S/N, 07738 México, DF, Mexico

Correspondence should be addressed to Jesús A. Meda Campaña; jmedac@ipn.mx

Received 10 January 2015; Revised 1 April 2015; Accepted 1 April 2015

Academic Editor: Qingling Zhang

Copyright (c) 2015 Tonatiuh Hernández-Cortés et al. This is an open access article distributed under the Creative Commons Attribution License, which permits unrestricted use, distribution, and reproduction in any medium, provided the original work is properly cited.

\begin{abstract}
This paper is devoted to solve the regulation problem on the basis of local regulators, which are combined using "new" membership functions. As a result, the exact tracking of references is achieved. The design of linear local regulators is suggested in this paper, but now adequate membership functions are computed in order to ensure the proper combination of the local regulators in the interpolation regions. These membership functions, which are given as mathematical expressions, solve the fuzzy regulation problem in a relative simple way. The form of the new membership functions is systematically derived for a class of Takagi-Sugeno (T-S) fuzzy systems. Some numerical examples are used to illustrate the viability of the proposed approach.
\end{abstract}

\section{Introduction}

One of the most important problems in control theory consists of finding a controller capable of taking the outputs of a plant towards the reference signals generated by an external system, named exosystem.

This problem has been studied by several authors due to its wide applicability in mechanical systems, aeronautics, and telematics, just to name a few.

The works of Francis [1] and Francis and Wonham [2] have shown that the solvability of a multivariable linear regulator problem corresponds to the solvability of a system of two linear matrix equations, called Francis Equations. Also they have shown that, in the case of error feedback, the regulator which solves the problem includes the exosystem. This property is commonly know as Internal Model Principle.

Later, Isidori, and Byrnes [3] showed that the result established by Francis could be extended to the nonlinear field as a general case and that the equations of Francis represent a particular case of a set of nonlinear equations. They showed that the solvability for the nonlinear case depends on the solution of a set of nonlinear partial differential equations, called Francis-Isidori-Byrnes (FIB) equations. Unfortunately, such equations are too difficult to solve in a practical manner, in general.

On the other hand, some techniques have been proven to be an alternative to solve this problem by combining the theory of output regulation and the Takagi-Sugeno fuzzy modeling [4-9].

In [10], the authors propose an approach based on the weighted summation of local linear regulators in order to synchronize chaotic systems by means of regulation theory. However, to ensure the exact output regulation, two conditions need to be fulfilled: (1) the same input matrix for all subsystems, that is, $B_{1}=B_{2}=\cdots=B_{r}$, where $r$ is the number of rules in the fuzzy model, and (2) same zero error manifold $\pi(w(t))$ for every local subsystem.

Later in [11], the exact output regulator is directly designed for the overall T-S fuzzy model. Although such a controller achieves the exact output regulation where the weighted summation of linear local regulators fails, its expression may be very large.

For that reason, in the present paper the simplicity of the fuzzy regulator obtained from the weighted summation of linear local regulators is exploited; the effectiveness of the controller given in this approach guarantees at least for a class 
of T-S fuzzy models the exact output regulation. To this end, new membership functions will be systematically computed in order to adequately combine the linear local regulators, guaranteeing in this way the proper performance of the fuzzy regulator in the interpolation regions. A preliminary result has been given in [6], where the new membership functions are approximated by soft computing techniques.

The main idea comes from the fact that each local controller is valid, at least, for its corresponding local system, while the fuzzy interpolation regions require more attention at the moment of evaluating the performance of the overall fuzzy controller. For that reason, the proposed approach consists of finding new membership functions capable of adequately combine adjacent local controllers in order to achieve the control goal.

So, the main contribution of the present work is to find a control law for a class of Takagi-Sugeno fuzzy models, in order to achieve exact output regulation on the basis of local regulators and computing of new membership functions, even if different input matrices appear in the linear local subsystems. Consequently, one of the restrictions given in [10] is avoided, and there is no need of verifying the existence of the fuzzy regulator for all $t \geq 0$ [11]. Besides, the new membership functions, allowing the proper combination the local regulators, are given as a mathematical expressions.

The rest of the paper is organized as follows. In Section 2 the nonlinear regulation problem formulation is given with a brief review of the Takagi-Sugeno models and the fuzzy regulation problem. The main result is developed in Section 3. In Section 4 some examples are presented and finally, in Section 5, some conclusions are drawn.

\section{The Output Regulation Problem}

Consider a nonlinear system defined by

$$
\begin{gathered}
\dot{x}(t)=f(x(t), \omega(t), u(t)), \\
y(t)=c(x(t)), \\
\dot{\omega}(t)=s(\omega(t)), \\
y_{\mathrm{ref}}(t)=q(\omega(t)), \\
e(t)=h(x(t), \omega(t)),
\end{gathered}
$$

where $x(t) \in \mathbb{R}^{n}$ is the state vector of the plant, $w(t) \in W \subset$ $\mathbb{R}^{s}$ is the state vector of the exosystem, which generates the reference and/or the perturbation signals, and $u(t) \in \mathbb{R}^{m}$ is the input signal. Equation (5) refers to difference between output system of the plant $\left(y(t) \in \mathbb{R}^{m}\right)$ and the reference signal $\left(y_{\text {ref }}(t) \in \mathbb{R}^{m}\right)$, that is, $h(x(t), \omega(t))=y(t)-y_{\text {ref }}=$ $c(x(t))-q(x(t))$, taking into account that $m \leq n$. Besides, it is assumed that $f(x, u, w), h(x, w)$ and $w(t)$ are $C^{k}$ functions (for some large $k$ ) of their arguments and also that $f(0,0,0)=$ $0, s(0)=0$, and $h(0,0)=0[12]$.
Clearly, by linearizing (1)-(5) around $x=0$ one gets

$$
\begin{gathered}
\dot{x}(t)=A x(t)+B u(t)+P w(t), \\
y(t)=C x(t), \\
\dot{w}(t)=S w(t), \\
y_{\text {ref }}(t)=Q w(t), \\
e(t)=C x(t)-Q w(t) .
\end{gathered}
$$

Thus, the Nonlinear Regulator Problem [3, 13] consists of finding a controller $u(t)=\alpha(x(t), w(t))$, such that the closedloop system, $\dot{x}(t)=A x(t)+B \alpha(x(t), 0)$, has an asymptotically stable equilibrium point, and the solution of the system (6) satisfies $\lim _{t \rightarrow \infty} e(t)=0$.

So, by defining $\pi(w(t))$ as the steady-state zero error manifold and $\gamma(w(t))$ as the steady-state input, the following theorem gives the conditions for the solution of nonlinear regulation problem.

Theorem 1. Suppose that $\dot{w}(t)=s(w(t))$ is Poisson stable and a gain $K$ exists such that the matrix $A+B K$ is stable and there exist mappings $x_{s s}(t)=\pi(w(t))$ and $u_{s s}=\gamma(w(t))$ with $\pi(0)=$ 0 and $\gamma(0)=0$ satisfying

$$
\begin{gathered}
\frac{\partial \pi(w(t))}{\partial w(t)} s(w(t))=f(\pi(w(t)), w(t), \gamma(w(t))), \\
0=h(\pi(w(t)), w(t)) .
\end{gathered}
$$

Then the signal control for the nonlinear regulation is given by

$$
u(t)=K(x(t)-\pi(w(t)))+\gamma(w(t)) .
$$

Proof. See $[12,13]$.

Nonlinear partial differential equations (7) are known as Francis-Isidori-Byrnes (FIB) equations and their linear counterparts are obtained when the mappings $x_{s s}(t)=$ $\pi(w(t))$ and $u_{s s}(t)=\gamma(w(t))$ become into $x_{s s}(t)=\Pi w(t)$ and $u_{s s}(t)=\Gamma w(t)$, respectively. Thus, the linear problem is reduced to solve a set of linear matrix equations (Francis equations) [1]:

$$
\begin{gathered}
\Pi S=A \Pi+B \Gamma+P, \\
0=C \Pi-Q .
\end{gathered}
$$

2.1. The Exact Output Fuzzy Regulation Problem. Takagi and Sugeno proposed a fuzzy model composed by a set of linear subsystem with IF-THEN rules capable of relating physical knowledge, linguistic characteristics, and properties of the system. Such a model successfully represents a nonlinear system at least in a predefined region of phase space [14]. It is important to remark that in this work the exosystem is purely "linear," because the computation of new membership functions for the general case is still an open problem.

The T-S model for system (1)-(5) is given by [15].

Model. Rule $i$ :

$$
\text { IF } z_{1}(t) \text { is } M_{1}^{i} \text { and } \cdots \text { and } z_{p}(t) \text { is } M_{p}^{i} \text {, }
$$


THEN

$$
\begin{array}{r}
\dot{x}(t)=A_{i} x(t)+B_{i} u(t)+P_{i} w(t), \\
\dot{w}(t)=S w(t), \\
e(t)=C_{i} x(t)-Q w(t), \\
i=1,2, \ldots, r,
\end{array}
$$

where $r$ is the number of rules in the model and $M_{j}^{i}$ is the fuzzy sets defined on the basis of the knowledge of the system.

Then, the regulation problem defined by (1)-(5) can be represented through the T-S fuzzy model; that is, [10]

$$
\begin{gathered}
\dot{x}(t)=\sum_{i=1}^{r} h_{i}(z(t))\left\{A_{i} x(t)+B_{i} u(t)+P_{i} w(t)\right\}, \\
\dot{w}(t)=S w(t), \\
e(t)=\sum_{k=1}^{r} h_{i}(z(t)) C_{i} x(t)-Q w(t),
\end{gathered}
$$

where $x(t) \in \mathbb{R}^{n}$ is the state vector of the plant, $w(t) \in \mathbb{R}^{s}$ is the state vector of the exosystem, $u(t) \in \mathbb{R}^{m}$ is the input signal, and $e(t) \in \mathbb{R}^{m}$ and $h_{i}(z(t))$ is the normalized weight of each rule, which depends on the membership function for the premise variable $z_{j}(t)$ in $M_{j}^{i}$, where

$$
\begin{gathered}
\bar{\omega}_{i}(z(t))=\prod_{j=1}^{p} M_{j}^{i}\left(z_{j}(t)\right), \\
h_{i}(z(t))=\frac{\bar{\omega}_{i}(z(t))}{\sum_{i=1}^{r} \bar{\omega}_{i}(z(t))}, \\
\sum_{i=1}^{r} h_{i}(z(t))=1, \\
h_{i}(z(t)) \geq 0,
\end{gathered}
$$

with $z(t)=\left[\begin{array}{llll}z_{1}(t) & z_{2}(t) & \cdots & z_{p}(t)\end{array}\right]$ as a function of $x(t), i=$ $1, \ldots, r$, and $j=1, \ldots, p$.

The Exact Fuzzy Regulator Problem consists of finding a controller $u(t)=\alpha(x(t), w(t))$, such that the closed-loop system,

$$
\dot{x}(t)=\sum_{i=1}^{r} h_{i}(z(t))\left\{A_{i} x(t)+B_{i} \alpha(x(t), 0)\right\}
$$

has an asymptotically stable equilibrium point, and the solution of system (11) satisfies $\lim _{t \rightarrow \infty} e(t)=0$.

From $[10,12,13]$ the desired overall fuzzy controller can be represented as

$$
u(t)=\sum_{h=1}^{r} h_{i}(z(t)) K_{i}[x(t)-\pi(w(t))]+\gamma(w(t)) .
$$

Considering that approximations for mappings $\pi(w(t))$ and $\gamma(w(t))$ can be obtained by

$$
\begin{gathered}
\tilde{\pi}(w(t))=\sum_{i=1}^{r} h_{i}(z(t)) \Pi_{i} w(t), \\
\tilde{\gamma}(w(t))=\sum_{i=1}^{r} h_{i}(z(t)) \Gamma_{i} w(t),
\end{gathered}
$$

then, the solution of the fuzzy regulation problem requires to obtain $\Pi_{i}$ and $\Gamma_{i}$ from the $r$ linear local regulators problems included in (11) and defined by

$$
\begin{gathered}
\Pi_{i} S=A_{i} \Pi_{i}+B_{i} \Gamma_{i}+P_{i}, \\
0=C_{i} \Pi_{i}-Q
\end{gathered}
$$

for $i=1, \ldots, r$.

Thus, the following controller is obtained:

$$
\begin{aligned}
u(t)= & \sum_{i=1}^{r} h_{i}(z(t)) K_{i}\left[x(t)-\sum_{i=1}^{r} h_{i}(z(t)) \Pi_{i} w(t)\right] \\
& +\sum_{i=1}^{r} h_{i}(z(t)) \Gamma_{i} w(t) .
\end{aligned}
$$

However, according to [10], the exact fuzzy regulation is achieved with this controller only when $\Pi_{1}=\cdots=\Pi_{r}=\Pi$, and

(1) input matrix is equal for all subsystems; that is, $B_{1}=$ $\cdots=B_{r}=B$, or

(2) the steady-state zero error manifold is equal for all subsystems; that is, $\Gamma_{1}=\cdots=\Gamma_{r}=\Gamma$.

In the present work, restrictions 1 and 2 are avoided by computing new membership functions for a class of T-S fuzzy systems. On the other hand, in [11] the Exact Output Fuzzy Regulation Problem (EOFRP) was solved by finding the steady-state zero error manifold $x_{s s}(t)=\pi(w(t))$ and the steady-state input $u_{s s}(t)=\gamma(w(t))$ for the overall T$S$ fuzzy problem through $e_{s s}(t)=x(t)-x_{s s}(t)=x(t)-$ $\pi(w(t))$ (steady-state error equation), resulting in $\pi(w(t))=$ $\Pi_{f}(t) w(t)$ and $\gamma(w(t))=\Gamma_{f}(t) w(t)$, where $\Pi_{f}(t)$ and $\Gamma_{f}(t)$ are continuous time-variant matrices and $\pi(w(t))=\Pi_{f}(t) w(t)$ is a $C^{k-1}$ function [12].

Within the next section, the exact output regulation, for a class of T-S fuzzy systems, is obtained by proposing a solution for $\gamma(w(t))$ on the basis of different membership functions in the regulator.

\section{The Exact Output Fuzzy Regulation by Local Regulators}

In this section, a particular class of T-S fuzzy models is considered to solve the exact output regulation on the basis of linear local controllers. So, the main goal is to find an overall regulator based on the fuzzy summation of local regulators considering adequate membership functions. Clearly, such 
membership functions are not necessarily the same included in the fuzzy plant. Thus, the steady-state input $\gamma(w(t))$ can be defined as

$$
\gamma(w(t))=\sum_{i=1}^{r} \mu_{i}(w(t)) \Gamma_{i} w(t)
$$

where $\mu_{i}(w(t))$ are new membership functions, such that the fuzzy output regulator obtained from local regulators coincides with the controller given in [11], at least for the class of fuzzy models considered. This novel approach only requires the computation of the linear local controllers and the computation of the new membership functions which will be presented below. The regulator obtained in this way is valid for all $t \geq 0$, while in [11] this condition needs to be verified.

Consider the following matrices:

$$
\begin{gathered}
A_{i}=\left[\begin{array}{ccccc}
0 & 1 & 0 & \cdots & 0 \\
0 & 0 & 1 & \cdots & 0 \\
\vdots & \vdots & \vdots & \ddots & \vdots \\
a_{1}^{i} & a_{2}^{i} & a_{3}^{i} & \cdots & a_{n}^{i}
\end{array}\right], \\
B_{i}=\left[\begin{array}{cc}
0 \\
0 \\
\vdots \\
s_{1}
\end{array}\right], \\
Q=\left[\begin{array}{ccccc}
0 & 1 & 0 & \cdots & 0 \\
0 & 0 & 1 & \cdots & 0 \\
\vdots & \vdots & \vdots & \ddots & \vdots \\
s_{1} & s_{2} & s_{3} & \cdots & s_{n}
\end{array}\right], \\
C_{i}=\left[\begin{array}{llll}
c_{1} & 0 & \cdots & 0
\end{array}\right], \\
Q=\left[\begin{array}{llll}
q_{1} & 0 & \cdots & 0
\end{array}\right],
\end{gathered}
$$

where $a_{j}^{i}$ represents the elements of the last row of $A_{i}$ and $b_{1}^{i}$ represents the element of the last row of $B_{i}$ with $j=1, \ldots, n$ and $i=1, \ldots, r$. It is worth mentioning that $A_{i}$ and $S$ are matrices of the same dimension, as shown in (19). Notice that matrices in the form of $S$ and $Q$ can be used to generate a great variety of signals, ensuring, in that way, the applicability of the approach in a great number of cases. Therefore, from (14) the control input can be defined by

$$
\begin{aligned}
u(t)= & \sum_{i=1}^{r} h_{i}(x(t)) k_{i}\{x(t)-\pi(w(t))\} \\
& +\sum_{i=1}^{r} \mu_{i}(w(t)) \Gamma_{i} w(t),
\end{aligned}
$$

because, as mentioned before, $z(t)$ is a function of $x(t)$ and in steady-state $x(t)=\pi(w(t))$.
On the other hand, from $C_{i}, Q$ and from (6), it can be concluded that $c_{1} x_{1}(t)=q_{1} w_{1}(t)$ in steady-state, while from (19) the following exosystem can be easily derived:

$$
\begin{aligned}
& \dot{w}_{1}=w_{2}, \\
& \dot{w}_{2}=w_{3}, \\
& \quad \vdots \\
& \dot{w}_{n}=s_{1} w_{1}+s_{2} w_{2}+\cdots+s_{n} w_{n}, \\
& \dot{w}_{n}=\sum_{i=1}^{n} s_{i} w_{i} .
\end{aligned}
$$

Besides, each subsystem can be rewritten as follows:

$$
\begin{aligned}
\dot{x}_{1} & =x_{2}, \\
\dot{x}_{2} & =x_{3}, \\
& \vdots \\
\dot{x}_{n} & =a_{1}^{i} x_{1}+a_{2}^{i} x_{2}+\cdots+a_{n}^{i} x_{n}+b_{1}^{i} u_{s s, i} .
\end{aligned}
$$

By equating the time derivative of the fuzzy output with the time derivative of the reference signal, it results in $c_{1} \dot{x}_{1}=$ $q_{1} \dot{w}_{1}$. Furthermore, from (21) and (22), it is possible to conclude that

$$
c_{1} \dot{x}_{i}=q_{1} \dot{w}_{i}
$$

where $i=1,2, \ldots, n$. From this analysis, two results can be obtained. First

$$
\Pi=\left[\begin{array}{cccc}
\frac{q_{1}}{c_{1}} & 0 & \cdots & 0 \\
0 & \frac{q_{1}}{c_{1}} & \cdots & 0 \\
\vdots & \vdots & \ddots & \vdots \\
0 & 0 & \cdots & \frac{q_{1}}{c_{1}}
\end{array}\right],
$$

and due to the shape of (19) $\Pi$ is constant and common for all subsystems. Thus, $\pi(w(t))=\Pi w(t)$. Second, the local steadystate input is defined by

$$
\begin{aligned}
u_{s s, i}= & \frac{\left(q_{1} / c_{1}\right) s_{1} w_{1}-\left(q_{1} / c_{1}\right) a_{1}^{i} w_{1}}{b_{1}^{i}} \\
& +\frac{\left(q_{1} / c_{1}\right) s_{2} w_{2}-\left(q_{1} / c_{1}\right) a_{2}^{i} w_{2}}{b_{1}^{i}} \\
& +\cdots+\frac{\left(q_{1} / c_{1}\right) s_{n} w_{n}-\left(q_{1} / c_{1}\right) a_{n}^{i} w_{n}}{b_{1}^{i}} .
\end{aligned}
$$


The previous expression can be rewritten in a compact form as follows:

$$
u_{s s, i}=\frac{\sum_{j=1}^{n}\left(q_{1} / c_{1}\right)\left[s_{j} w_{j}-a_{j}^{i} w_{j}\right]}{b_{1}^{i}} \quad \forall i=1, \ldots, r
$$

and from (18) the fuzzy steady-state input is

$$
\begin{aligned}
u_{s s}(t) & =\sum_{i=1}^{r} \mu_{i}(w(t)) u_{s s, i} \\
& =\sum_{i=1}^{r} \mu_{i}(w(t)) \Gamma_{i} w(t), \quad \forall i=1, \ldots, r .
\end{aligned}
$$

From the previous analysis, each $\Gamma_{i}$ can be computed from

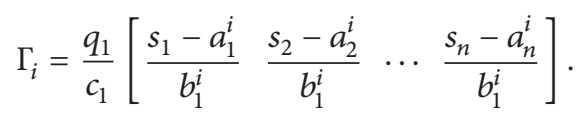

Now, it is possible to rewrite the fuzzy model as [11]

$$
\begin{gathered}
\dot{x}(t)=\widetilde{A}(t) x(t)+\widetilde{B}(t) u(t)+\widetilde{P}(t) w(t), \\
\dot{w}(t)=S w(t), \\
e(t)=\widetilde{C}(t) x(t)-Q w(t),
\end{gathered}
$$

where

$$
\begin{aligned}
& \widetilde{A}(t)=\sum_{i=1}^{r} h_{i}(z(t)) A_{i}, \\
& \widetilde{B}(t)=\sum_{i=1}^{r} h_{i}(z(t)) B_{i}, \\
& \widetilde{P}(t)=\sum_{i=1}^{r} h_{i}(z(t)) P_{i}, \\
& \widetilde{C}(t)=\sum_{i=1}^{r} h_{i}(z(t)) C_{i} .
\end{aligned}
$$

For the sake of simplicity, in the following analysis it is considered that $P_{i}=0, i=1, \ldots, r$, that is, $\widetilde{P}(t)=0$. However, taking similar steps as those used below, the result can be easily extended to the case involving $\widetilde{P}(t) \neq 0$.

Thus, from (19) and (29) it can be observed that the last equation $\dot{x}_{n}$ in (29) is given by

$$
\begin{aligned}
\dot{x}_{n}=[ & h_{1}(x(t))\left(a_{1}^{i} x_{1}+\cdots+a_{n}^{i} x_{n}\right) \\
& \left.+\cdots+h_{r}(x(t))\left(a_{1}^{i} x_{1}+\cdots+a_{n}^{i} x_{n}\right)\right] \\
+ & {\left[h_{1}(x(t)) b_{1}^{i}+\cdots+h_{r}(x(t)) b_{1}^{r}\right] u_{s s}(t), }
\end{aligned}
$$

where compact form is

$$
\dot{x}_{n}=\sum_{i=1}^{r} h_{i}(x(t))\left(\sum_{j=1}^{n} a_{j}^{i} x_{j}\right)+\left(\sum_{i=1}^{r} h_{i}(x(t)) b_{1}^{i}\right) u_{s s}(t),
$$

considering that in steady-state $x(t)=\Pi w(t)$, the total steady-state input can be obtained from (23), resulting in

$$
\begin{aligned}
\sum_{i=1}^{r} h_{i} & (w(t))\left(\frac{q_{1}}{c_{1}} \sum_{j=1}^{n} a_{j}^{i} w_{j}\right) \\
& +\left(\sum_{i=1}^{r} h_{i}(w(t)) b_{1}^{i}\right) u_{s s}(t)=\frac{q_{1}}{c_{1}} \sum_{i=1}^{n} s_{i} w_{i} .
\end{aligned}
$$

Consequently, the steady-state input for the total fuzzy system can be constructed by

$$
\begin{aligned}
& u_{s s}(t) \\
& =\frac{\left(q_{1} / c_{1}\right) \sum_{i=1}^{n} s_{i} w_{i}-\left(q_{1} / c_{1}\right) \sum_{i=1}^{r} h_{i}(w(t))\left(\sum_{j=1}^{n} a_{j}^{i} w_{j}\right)}{\sum_{i=1}^{r} h_{i}(w(t)) b_{1}^{i}} .
\end{aligned}
$$

Now, a new set of fuzzy membership functions $\mu_{i}(w(t))$, satisfying $\sum_{i=1}^{r} \mu_{i}(w(t))=1$ with $\mu_{i}(w(t)) \geq 0, i=1, \ldots, r$, will be computed in order to obtain a similar steady-state input to (36) but formed from the fuzzy summation of local linear regulators. At this point, the local regulators and total regulator are defined as follows:

$$
\begin{aligned}
u_{s s, i} & =\gamma(w(t))=\sum_{i=1}^{r} \mu_{i}(w(t)) \Gamma_{i} w(t) \\
& =\sum_{i=1}^{r} \mu_{i}(w(t)) \frac{\sum_{j=1}^{n}\left(q_{1} / c_{1}\right)\left[s_{j} w_{j}-a_{j}^{i} w_{j}\right]}{b_{1}^{i}}, \\
u_{s s} & =\gamma(w(t)) \\
& =\frac{\left(q_{1} / c_{1}\right) \sum_{i=1}^{n} s_{i} w_{i}-\left(q_{1} / c_{1}\right) \sum_{i=1}^{r} h_{i}(w(t))\left(\sum_{j=1}^{n} a_{j}^{i} w_{j}\right)}{\sum_{i=1}^{r} h_{i}(w(t)) b_{1}^{i}},
\end{aligned}
$$

respectively. Thus, the local fuzzy regulators will be multiplied by the new membership functions and the result will be equated to the global fuzzy regulator (36) as follows:

$$
\begin{gathered}
\frac{\left(q_{1} / c_{1}\right) \sum_{i=1}^{n} s_{i} w_{i}-\left(q_{1} / c_{1}\right) \sum_{i=1}^{r} h_{i}(w(t))\left(\sum_{j=1}^{n} a_{j}^{i} w_{j}\right)}{\sum_{i=1}^{r} h_{i}(w(t)) b_{1}^{i}} \\
=\sum_{i=1}^{r} \mu_{i}(w(t)) \frac{\sum_{j=1}^{n}\left(q_{1} / c_{1}\right)\left[s_{j} w_{j}-a_{j}^{i} w_{j}\right]}{b_{1}^{i}} .
\end{gathered}
$$

So, the adequate membership functions are

$$
\mu_{i}(w(t))=\frac{b_{1}^{i} h_{i}(w(t))}{\sum_{i=1}^{r} h_{i}(w(t)) b_{1}^{i}},
$$

with $i=1,2, \ldots, r$. It is important to remark that the new membership functions $\mu_{i}(w(t))$ are given in terms of $h_{i}(w(t))$ for simplicity. However, $h_{i}(w(t))$ can be removed from the new membership functions (39), when $h_{i}(w(t))$ is replaced by its corresponding expression. Besides, $h_{i}(w(t))$ is directly obtained from $h_{i}(x(t))$ by considering that in steady-state $x(t)=\Pi w(t)$. 
Notice that (39) fulfills conditions

$$
\begin{gathered}
\sum_{i=1}^{r} \mu_{i}(w(t))=1, \\
\mu_{i}(w(t)) \geq 0,
\end{gathered}
$$

for all $i=1,2, \ldots, r$.

Remark 2. It can be observed that (39) is always valid when the entries of input matrix $b_{1}^{i}$ have the same sign; if this condition is not fulfilled the denominators of the new fuzzy membership functions will present singularities. Nevertheless, these singularities may appear outside the operation region, allowing more flexibility, but such cases require a particular study of the system, which is beyond the scope of this work.

The following theorem provides the conditions for the existence of the exact output fuzzy regulator for a class of T-S fuzzy models.

Theorem 3. The exact fuzzy output regulation with full information for T-S fuzzy systems in the form of (11), defined by matrices (19), is solvable if (a) the sign of entries for corresponding position, inside input matrices $B_{i}$ for $i=$ $1, \ldots, r$, is the same, (b) there exists a fuzzy stabilizer $u(t)=$ $\sum_{i=1}^{r} h_{i}(z(t)) K_{i} x$ for the T-S fuzzy system [16], and (c) the exosystem $\dot{w}(t)=S w(t)$ is Poisson stable. Moreover, the Exact Output Fuzzy Regulation Problem is solvable by the controller:

$$
\begin{aligned}
u(t)= & \sum_{i=1}^{r} h_{i}(x(t)) k_{i}\{x(t)-\Pi w(t)\} \\
& +\sum_{i=1}^{r} \mu_{i}(w(t)) \Gamma_{i} w(t)
\end{aligned}
$$

where $\mu_{i}(w(t))$ can be readily obtained from (39).

Proof. From the previous analysis, the existence of mappings $\pi(w(t))=\Pi w(t)$ and $\gamma(w(t))=\sum_{i=1}^{r} \mu_{i}(w(t)) \Gamma_{i} w(t)$ is guaranteed when the local subsystems are defined by (19); that is, the form of $\pi(w(t))$ corresponds to a diagonal matrix and will be the same for all subsystems, while $\gamma(w(t))$ can be directly substitute.

On the other hand, condition (a) avoids singularities in the new membership functions $\mu_{i}(w(t))$, while the inclusion of condition (b) has been thoroughly discussed in $[12,13,17-$ 19], and it implies that the steady-state manifold can be made asymptotically stable by the action of the fuzzy stabilizer.

Finally, condition (c) is introduced to avoid that the reference signal converges to zero, because if the reference tends to zero, then the regulation problem turns into a stabilization one, which can be solved by means of the fuzzy stabilizer. In addition, as mentioned before, this kind of matrix $S$ can be used to generate a great number of reference signals. The rest of the proof follows directly from the previous analysis.

Remark 4. Although, the PDC approach [14] can be used to compute the fuzzy stabilizer, more relaxed approaches can be considered to verify condition (b). For instance [16, 20-25].

\section{Exact Output Fuzzy Regulation Problem for a Class of T-S Fuzzy Systems with Multiple Inputs and Multiple Outputs (MIMO Case)}

Now the problem will be extended to T-S fuzzy MIMO models defined by matrices:

$$
\begin{aligned}
& A_{i}=\left[\begin{array}{c}
O \mid I \\
\hline D_{i}
\end{array}\right], \quad B_{i}=\left[\begin{array}{c}
O \\
F_{i}
\end{array}\right],
\end{aligned}
$$

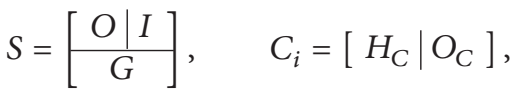

$$
\begin{aligned}
& Q_{i}=\left[H_{Q} \mid O_{Q}\right] \text {, }
\end{aligned}
$$

for $i=1, \ldots, r$, with

$$
\begin{aligned}
& I=\left[\begin{array}{cccc}
1 & 0 & \cdots & 0 \\
0 & 1 & \cdots & 0 \\
\vdots & \vdots & \ddots & \vdots \\
0 & 0 & \cdots & 1
\end{array}\right] \\
& O=\left[\begin{array}{cccc}
0 & 0 & \cdots & 0 \\
0 & 0 & \cdots & 0 \\
\vdots & \vdots & \ddots & \vdots \\
0 & 0 & \cdots & 0
\end{array}\right] \\
& D_{i}=\left[\begin{array}{ccccc}
a_{(k+1), 1}^{i} & a_{(k+1), 2}^{i} & a_{(k+1), 3}^{i} & \cdots & a_{(k+1), n}^{i} \\
a_{(k+2), 1}^{i} & a_{(k+2), 2}^{i} & a_{(k+2), 3}^{i} & \cdots & a_{(k+2), n}^{i} \\
\vdots & \vdots & \vdots & \ddots & \vdots \\
a_{n, 1}^{i} & a_{n, 2}^{i} & a_{n, 3}^{i} & \cdots & a_{n, n}^{i}
\end{array}\right] \text {, } \\
& F_{i}=\left[\begin{array}{cccc}
b_{k+1}^{i} & 0 & \cdots & 0 \\
0 & b_{k+2}^{i} & \cdots & 0 \\
\vdots & \vdots & \ddots & \vdots \\
0 & 0 & \cdots & b_{n}^{i}
\end{array}\right] \text {, } \\
& G=\left[\begin{array}{ccccc}
s_{(k+1), 1} & s_{(k+1), 2} & s_{(k+1), 3} & \cdots & s_{(k+1), n} \\
s_{(k+2), 1} & s_{(k+2), 2} & s_{(k+2), 3} & \cdots & s_{(k+2), n} \\
\vdots & \vdots & \vdots & \ddots & \vdots \\
s_{n, 1} & s_{n, 2} & s_{n, 3} & \cdots & s_{n, n}
\end{array}\right] \text {, } \\
& O_{C}=O_{Q}=\left[\begin{array}{cccc}
0 & 0 & \cdots & 0 \\
0 & 0 & \cdots & 0 \\
\vdots & \vdots & \ddots & \vdots \\
0 & 0 & \cdots & 0
\end{array}\right]
\end{aligned}
$$




$$
\begin{aligned}
H_{C} & =\left[\begin{array}{cccc}
c_{1} & 0 & \cdots & 0 \\
0 & c_{2} & \cdots & 0 \\
\vdots & \vdots & \ddots & \vdots \\
0 & 0 & \cdots & c_{m}
\end{array}\right], \\
H_{Q} & =\left[\begin{array}{cccc}
q_{1} & 0 & \cdots & 0 \\
0 & q_{2} & \cdots & 0 \\
\vdots & \vdots & \ddots & \vdots \\
0 & 0 & \cdots & q_{m}
\end{array}\right],
\end{aligned}
$$

where $k=n-m, F_{i} \in \mathbb{R}^{m \times m}, O \in \mathbb{R}^{(n-m) \times m}, O_{C} \in \mathbb{R}^{m \times m}$, $I \in \mathbb{R}^{(n-m) \times(n-m)}, D_{i} \in \mathbb{R}^{m \times n}, H_{C} \in \mathbb{R}^{m \times m}, H_{Q} \in \mathbb{R}^{m \times m}$, and $G \in \mathbb{R}^{m \times n}$. As before, this proposal is given because a great number of mechanical systems can be represented in this form.

Notice that this analysis cannot be omitted because the computation of the new membership functions is not the same as that developed in the previous section. From (42) the following exosystem can be easily derived:

$$
\begin{aligned}
& \dot{w}_{1}=w_{m+1}, \\
& \dot{w}_{2}=w_{m+2}, \\
& \vdots \\
& \dot{w}_{k+1}=s_{(k+1), 1} w_{1}+s_{(k+1), 2} w_{2}+\cdots+s_{(k+1), n} w_{n} \\
& \quad=\sum_{j=1}^{n} s_{(k+1), j} w_{j}, \\
& \dot{w}_{k+2}=s_{(k+2), 1} w_{1}+s_{(k+2), 2} w_{2}+\cdots+s_{(k+2), n} w_{n} \\
& \quad=\sum_{j=1}^{n} s_{(k+2), j} w_{j}, \\
& \vdots \\
& \dot{w}_{n}=s_{n, 1} w_{1}+s_{n, 2} w_{2}+\cdots+s_{n, n} w_{n}=\sum_{j=1}^{n} s_{n, j} w_{j} .
\end{aligned}
$$

Moreover, each subsystem can be described by

$$
\begin{aligned}
& \dot{x}_{1}=x_{m+1} \text {, } \\
& \dot{x}_{2}=x_{m+2} \text {, } \\
& \text { : } \\
& \dot{x}_{k+1}=a_{(k+1), 1}^{i} x_{1}+a_{(k+1), 2}^{i} x_{2}+\cdots+a_{(k+1), n} x_{n} \\
& +b_{k+1}^{i} u_{s s}^{i} \\
& =\sum_{j=1}^{n} a_{(k+1), j}^{i} x_{j}+b_{k+1}^{i} u_{s s}^{i}, \\
& \dot{x}_{k+2}=a_{(k+2), 1}^{i} x_{1}+a_{(k+2), 2}^{i} x_{2}+\cdots+a_{(k+2), n} x_{n} \\
& +b_{k+2}^{i} u_{s s}^{i} \\
& =\sum_{j=1}^{n} a_{(k+2), j}^{i} w_{j}+b_{1+2}^{i} u_{s s}^{i}, \\
& \dot{x}_{n}=s_{n, 1} w_{1}+s_{n, 2} w_{2}+\cdots+s_{n, n} w_{n}+b_{n}^{i} u_{s s}^{i} \\
& =\sum_{j=1}^{n} s_{n, j} w_{j}+b_{n}^{i} u_{s s}^{i} .
\end{aligned}
$$

As before, using expressions (44) and (45), considering $c_{1} x_{1}=$ $q_{1} w_{1}, c_{2} x_{2}=q_{2} w_{2}, \ldots, c_{m} x_{m}=q_{m} w_{m}$, and performing successive substitutions of $\dot{x}_{\ell}=\pi_{\ell} \dot{w}_{\ell}$ with $\ell=1, \ldots, m$, the following results are obtained:

$$
\Pi=\left[\begin{array}{cccc}
\pi_{1} & 0 & \cdots & 0 \\
0 & \pi_{2} & \cdots & 0 \\
\vdots & \vdots & \ddots & \vdots \\
0 & 0 & \cdots & \pi_{n}
\end{array}\right],
$$

where $\Pi \in \mathbb{R}^{n \times n}$, and $i=1,2, \ldots, r$; also $\pi_{1}=$ $q_{1} / c_{1}, \pi_{2}=q_{2} / c_{2}, \ldots, \pi_{m}=q_{m} / c_{m}, \pi_{m+1}=q_{1} / c_{1}, \pi_{m+2}=$ $q_{2} / c_{2}, \ldots, \pi_{m+m}=q_{m} / c_{m}$, and so forth. Notice that the values of $\pi_{*}$ repeat themselves every $m$ entry up to $n$. Additionally, $\Pi$ is constant and common for all subsystems while the local steady-state inputs are defined by

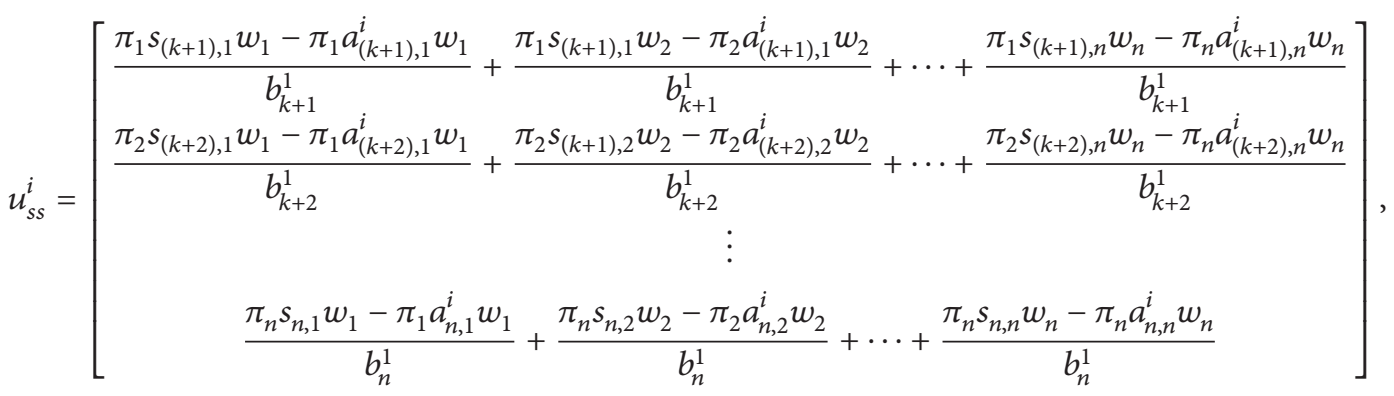


for $i=1, \ldots, r$. In compact form, (47) turns into

$$
u_{s s}^{i}=\left[\begin{array}{c}
\sum_{j=1}^{n} \frac{\pi_{1} s_{(k+1), j} w_{j}-\pi_{j} a_{(k+1), j} w_{j}}{b_{k+1}^{i}} \\
\sum_{j=1}^{n} \frac{\pi_{2} s_{(k+2), j} w_{j}-\pi_{j} a_{(k+2), j} w_{j}}{b_{k+2}^{i}} \\
\vdots \\
\sum_{j=1}^{n} \frac{\pi_{n} s_{n, j} w_{j}-\pi_{j} a_{n, j} w_{j}}{b_{n}^{i}}
\end{array}\right]
$$

and from (27) the local matrices $\Gamma_{i}$ have the form

$$
\Gamma_{i}=\left[\begin{array}{cccc}
\frac{\pi_{1} s_{(k+1), 1}-\pi_{1} a_{(k+1), 1}^{i}}{b_{k+1}^{i}} & \frac{\pi_{1} s_{(k+1), 2}-\pi_{2} a_{(k+1), 2}^{i}}{b_{k+1}^{i}} & \cdots & \frac{\pi_{1} s_{n, 1}-\pi_{n} a_{(k+1), n}^{i}}{b_{k+1}^{i}} \\
\frac{\pi_{2} s_{(k+2), 1}-\pi_{1} a_{(k+2), 1}^{i}}{b_{k+2}^{i}} & \frac{\pi_{2} s_{(k+2), 2}-\pi_{2} a_{(k+2), 2}^{i}}{b_{k+2}^{i}} & \cdots & \frac{\pi_{2} s_{n, 1}-\pi_{n} a_{(k+2), n}^{i}}{b_{k+2}^{i}} \\
\vdots & \vdots & \ddots & \vdots \\
\frac{\pi_{n} s_{n, 1}-\pi_{1} a_{n, 1}^{i}}{b_{n}^{i}} & \frac{\pi_{n} s_{n, 2}-\pi_{2} a_{n, 2}^{i}}{b_{n}^{i}} & \cdots & \frac{\pi_{n} s_{n, 1}-\pi_{n} a_{n, n}^{i}}{b_{n}^{i}}
\end{array}\right] .
$$

Now, again from the fuzzy plant given in (42) and the global fuzzy model (29), the expressions for $\dot{x}_{k+1}, \ldots, \dot{x}_{n}$ are

$$
\begin{aligned}
& \dot{x}_{k+1}= \sum_{i=1}^{r} h_{i}(x(t))\left(\sum_{j=1}^{n} a_{(k+1), j}^{i} x_{j}\right) \\
&+\left(\sum_{i=1}^{r} h_{i}(x(t)) b_{k+1}^{i}\right) u_{s s} \\
& \dot{x}_{k+2}= \sum_{i=1}^{r} h_{i}(x(t))\left(\sum_{j=1}^{n} a_{(k+2), j}^{i} x_{j}\right) \\
&+\left(\sum_{i=1}^{r} h_{i}(x(t)) b_{k+2}^{i}\right) u_{s s} \\
& \vdots+\left(\sum_{i=1}^{r} h_{i}(x(t)) b_{n}^{i}\right) u_{s s} . \\
& \dot{x}_{n}=\sum_{i=1}^{r} h_{i}(x(t))\left(\sum_{j=1}^{n} a_{n, j}^{i} x_{j}\right)
\end{aligned}
$$

So, by applying successive substitutions as before with (44), (45) and knowing that in steady-state $x_{j}=\pi_{j} w_{j}$, the following expressions are obtained:

$$
\begin{aligned}
& \sum_{i=1}^{r} h_{i}(w(t))\left(\sum_{j=1}^{n} a_{(k+1), j}^{i} \pi_{j} w_{j}\right) \\
& +\left(\sum_{i=1}^{r} h_{i}(w(t)) b_{k+1}^{i}\right) u_{s s}=\pi_{1} \sum_{i=1}^{n} s_{(k+1), i} w_{i} \\
& \sum_{i=1}^{r} h_{i}(w(t))\left(\sum_{j=1}^{n} a_{(k+2), j}^{i} \pi_{j} w_{j}\right) \\
& +\left(\sum_{i=1}^{r} h_{i}(w(t)) b_{k+2}^{i}\right) u_{s s}=\pi_{2} \sum_{i=1}^{n} s_{(k+2),} i w_{i}
\end{aligned}
$$

$$
\begin{aligned}
\sum_{i=1}^{r_{1}} h_{i}( & w(t))\left(\sum_{j=1}^{n} a_{n, j}^{i} \pi_{j} w_{j}\right) \\
& +\left(\sum_{i=1}^{r} h_{i}(w(t)) b_{n}^{i}\right) u_{s s}=\pi_{n} \sum_{i=1}^{n} s_{n, i} w_{i} .
\end{aligned}
$$


Therefore, the steady-state input for the overall fuzzy system is

$$
\begin{aligned}
& u_{s s} \\
& =\left[\begin{array}{c}
\frac{\pi_{1} \sum_{i=1}^{n} s_{(k+1), i} w_{i}-\sum_{i=1}^{r} h_{i}(w(t))\left(\sum_{j=1}^{n} a_{(k+1), j}^{i} \pi_{j} w_{j}\right)}{\sum_{i=1}^{r} h_{i}(w(t)) b_{k+1}^{i}} \\
\pi_{2} \sum_{i=1}^{n} s_{(k+2), i} w_{i}-\sum_{i=1}^{r} h_{i}(w(t))\left(\sum_{j=1}^{n} a_{(k+2), j}^{i} \pi_{j} w_{j}\right) \\
\sum_{i=1}^{r} h_{i}(w(t)) b_{k+2}^{i} \\
\vdots \\
\frac{\pi_{n} \sum_{i=1}^{n} s_{n, j} w_{i}-\sum_{i=1}^{r} h_{i}(w(t))\left(\sum_{j=1}^{n} a_{n, j}^{i} \pi_{j} w_{j}\right)}{\sum_{i=1}^{r} h_{i}(w(t)) b_{n}^{i}}
\end{array}\right] .
\end{aligned}
$$

But, the desired form of the steady-state is

$$
u_{s s}(t)=\gamma(w(t))=\sum_{i=1}^{r} \mu_{i}(w(t)) \Gamma_{i} w(t)
$$

Consequently, the new membership functions, $\mu_{i}(w(t))$, can be obtained after equating (52) and (53), resulting in

$$
\mu_{i}(w(t))=\left[\begin{array}{cccc}
\mu_{i, 1,1} & 0 & \cdots & 0 \\
0 & \mu_{i, 2,2} & \cdots & 0 \\
\vdots & \vdots & \ddots & \vdots \\
0 & 0 & \cdots & \mu_{i, m, m}
\end{array}\right]
$$

where

$$
\mu_{i, j, l}=\frac{b_{k+j}^{i} h_{i}(w(t))}{\sum_{i=1}^{r} h_{i}(w(t)) b_{k+j}^{i}}
$$

for all $i=1, \ldots, r ; j=l=1, \ldots, m$. It can be readily observed that the sum of the corresponding elements is equal to one and $\mu_{i, j, l}(w(t)) \geq 0$.

These new membership functions $\mu_{i}(w(t))$ are organized in a matrix form; for that reason $\sum_{i=1}^{r} \mu_{i}(w(t))=I$ (where $I$ is the identity matrix), the arrangement of (54) results as a consequence of ensuring (20) and again this representation is valid when the values of the input matrices $B_{i}$ have the same sign at corresponding positions. Clearly, this condition avoids singularities in (55). As before, $h_{i}(w(t))$ is directly obtained from $h_{i}(x(t))$ by considering that in steady-state $x(t)=\Pi w(t)$. At this point, the following theorem can be defined.

Theorem 5. The exact fuzzy output regulation with full information for T-S fuzzy MIMO systems in the form of (11), defined by matrices (42), is solvable if (a) the sign of entries for corresponding position, inside input matrices $B_{i}$ for $i=1, \ldots, r$, is the same, (b) there exists a fuzzy stabilizer $u(t)=\sum_{i=1}^{r} h_{i}(z(t)) K_{i} x$ for the fuzzy system [16], and (c) the exosystem $\dot{w}(t)=S w(t)$ is Poisson stable.
Moreover, the Exact Output Fuzzy Regulation Problem for MIMO systems is solvable by the controller:

$$
\begin{aligned}
u(t)= & \sum_{i=1}^{r} h_{i}(x(t)) k_{i}\{x(t)-\Pi w(t)\} \\
& +\sum_{i=1}^{r} \mu_{i}(w(t)) \Gamma_{i} w(t),
\end{aligned}
$$

where $\mu_{i}(w(t))$ can be readily obtained from (54) and (55).

Proof. It follows directly from Theorem 3 and previous analysis.

Remark 6. Notice that $\mu_{i}(w(t))$ is defined as a matrix and this is not a typical membership function; however, $\mu_{i}(w(t))$ contains a set of membership functions related to each entry of $B_{i}$. This form allows us to design controller (20).

\section{Numerical Examples}

5.1. Simple Input Case. Consider the problem of balancing and swing of an inverted pendulum on a cart. The motion equations for this system are [14]

$$
\begin{gathered}
\dot{x}_{1}(t)=x_{2}(t), \\
\dot{x}_{2}(t) \\
=\frac{g \sin \left(x_{1}(t)\right)-a m l x_{2}^{2} \sin \left(2 x_{1}(t)\right) / 2-a \cos \left(x_{1}(t)\right) u(t)}{4 l / 3-a m l \cos ^{2}\left(x_{1}(t)\right)}
\end{gathered}
$$

where $x_{1}(t)$ denotes the angle (in radians) of the pendulum from the vertical and $x_{2}(t)$ is the angular velocity; $g=$ $9.8 \mathrm{~m} / \mathrm{s}^{2}$ is the gravity constant, $m$ is the mass of the pendulum, $M$ is the mass of the cart, $2 l$ is the length of the pendulum, and $u$ is the force applied to the cart (in newtons); $a=1 /(m+M)$, with $m=2.0 \mathrm{~kg}, M=8.0 \mathrm{~kg}$, and $2 l=1.0 \mathrm{~m}$ in the simulations. The goal is to track the reference defined by the exosystem within the range $x_{1} \in(-\pi / 2, \pi / 2)$. To this end, a fuzzy model representing the nonlinear dynamics is as follows.

Rule 1.

IF $x_{1}(t)$ is about 0 ,

THEN $\dot{x}(t)=A_{1} x(t)+B_{1} u(t)$.

Rule 2.

IF $x_{1}(t)$ is about $\pm \pi / 2\left(\left|x_{1}\right|<\pi / 2\right)$,

THEN $\dot{x}(t)=A_{2} x(t)+B_{2} u(t)$. 


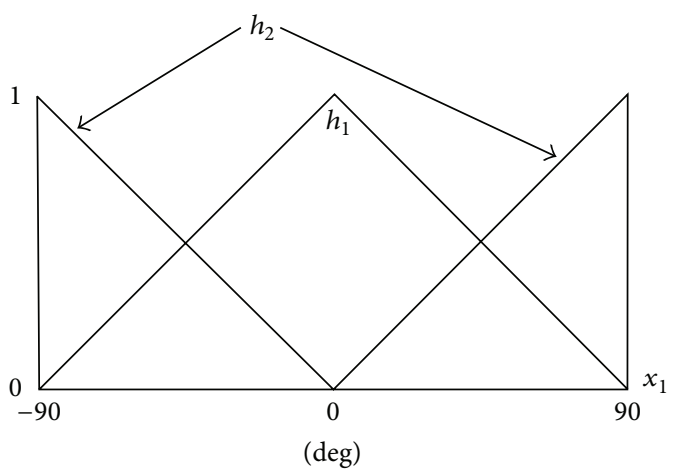

FIGURE 1: Membership functions of two-rule model.

Membership functions for Rules 1 and 2 are shown in Figure 1. In this example, the T-S fuzzy model of the plant is defined by matrices:

$$
\begin{gathered}
A_{1}=\left[\begin{array}{cc}
0 & 1 \\
17.2941 & 0
\end{array}\right], \quad B_{1}=\left[\begin{array}{c}
0 \\
-0.1765
\end{array}\right], \\
A_{2}=\left[\begin{array}{cc}
0 & 1 \\
4.1585 & 0
\end{array}\right], \quad B_{2}=\left[\begin{array}{c}
0 \\
-0.1500
\end{array}\right], \\
C_{1}=C_{2}=\left[\begin{array}{ll}
1 & 0
\end{array}\right],
\end{gathered}
$$

while the exosystem can be constructed from the following matrices:

$$
S=\left[\begin{array}{cc}
0 & 1 \\
-1 & 0
\end{array}\right], \quad Q=\left[\begin{array}{ll}
1 & 0
\end{array}\right]
$$

which allows us to generate a great kind of sinusoidal references. Figure 2 shows the simulation results after applying the controller:

$$
\begin{aligned}
u(t)= & \sum_{i=1}^{r} h_{i}(x(t)) K_{i}\left[x(t)-\sum_{i=1}^{r} h_{i}(w(t)) \Pi_{i} w(t)\right] \\
& +\sum_{i=1}^{r} h_{i}(w(t)) \Gamma_{i} w(t),
\end{aligned}
$$

defined in [10]. As expected, controller (61) is unable to achieve exact regulation, although tracking error remains bounded.

Now, using the method derived in this work, the local matrices $\Gamma_{i}$ can be readily obtained from (28), resulting in

$$
\Gamma_{1}=\left[\begin{array}{ll}
103.6667 & 0
\end{array}\right], \quad \Gamma_{2}=\left[\begin{array}{ll}
34.3836 & 0
\end{array}\right] .
$$

Besides, knowing that $q_{1} / c_{1}=1$, matrix $\Pi$ is obtained from (24):

$$
\Pi=\left[\begin{array}{ll}
1 & 0 \\
0 & 1
\end{array}\right]
$$

while the new membership functions computed from (39) are

$$
\begin{aligned}
& \mu_{1}(w(t))=\frac{\left(h_{1}(w(t))\right)(-0.1765)}{h_{1}(w(t))(-0.1765)+h_{2}(w(t))(-0.1500)}, \\
& \mu_{2}(w(t))=\frac{\left(h_{2}(w(t))\right)(-0.1500)}{h_{1}(w(t))(-0.1765)+h_{2}(w(t))(-0.1500)} .
\end{aligned}
$$

On the other hand, the fuzzy stabilizer for this system is constructed by means of the PDC approach developed in [14], which is formed by

$$
K_{1}=\left[\begin{array}{ll}
123.4898 & 6.9979
\end{array}\right], \quad K_{2}=\left[\begin{array}{lll}
58.6405 & 7.7962
\end{array}\right] .
$$

Figure 3 shows simulation with the controller defined in (20). Observe how the new membership functions are capable of combining the local regulators in the proper way to accomplish asymptotic tracking.

To verify the effectiveness of the latter regulator, the fuzzy controller is applied on the original system (57). The simulation results appear in Figure 4. Notice that the tracking error depicted in Figure 4(b) is due to the approximation provided by the T-S fuzzy model. Such an error can be reduced by considering a better approximation or an exact representation of the nonlinear system, as the one given by the nonlinear sector approach [14].

5.2. Multiple Inputs and Multiple Outputs (MIMO) Case. Now, consider a T-S fuzzy system of two rules, defined by the following matrices:

$$
A_{1}=\left[\begin{array}{llll}
0 & 0 & 1 & 0 \\
0 & 0 & 0 & 1 \\
6 & 4 & 2 & 4 \\
2 & 3 & 1 & 5
\end{array}\right],
$$

$$
B_{1}=\left[\begin{array}{cc}
0 & 0 \\
0 & 0 \\
-7 & 0 \\
0 & 2
\end{array}\right] \text {, }
$$

$$
A_{2}=\left[\begin{array}{llll}
0 & 0 & 1 & 0 \\
0 & 0 & 0 & 1 \\
3 & 4 & 2 & 1 \\
2 & 4 & 6 & 2
\end{array}\right],
$$

$$
\begin{gathered}
B_{2}=\left[\begin{array}{cc}
0 & 0 \\
0 & 0 \\
-3 & 0 \\
0 & 5
\end{array}\right], \\
C_{1}=C_{2}=\left[\begin{array}{llll}
1 & 0 & 0 & 0 \\
0 & 1 & 0 & 0
\end{array}\right],
\end{gathered}
$$




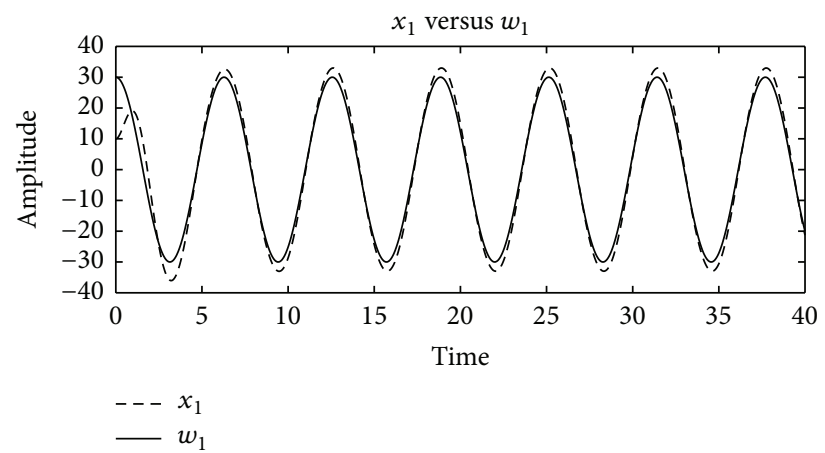

(a)

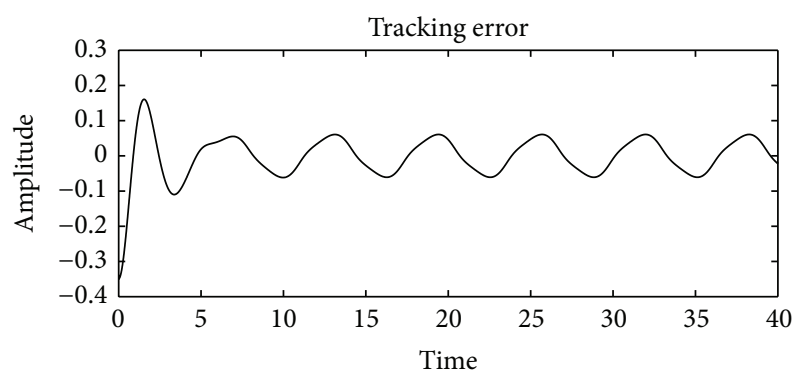

$-e$

(b)

FiguRE 2: (a) Output versus reference and (b) tracking error. When the fuzzy controller is designed on the basis of linear regulators and the fuzzy memberships of the plant are considered in the construction the fuzzy regulator.

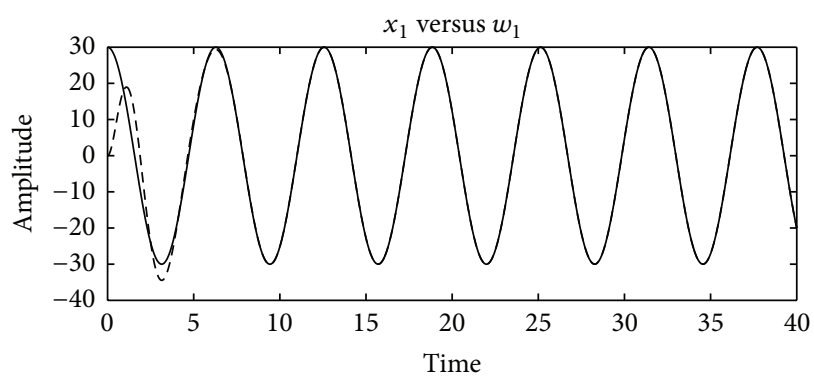

$---x_{1}$

$-w_{1}$

(a)

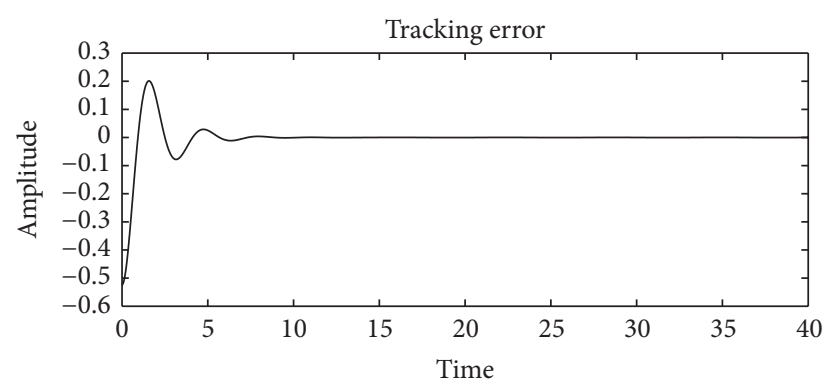

$-e$

(b)

FiguRE 3: (a) Output reference and (b) tracking error. When the fuzzy controller is designed on local regulators and the adequate membership functions for the fuzzy regulator are computed.

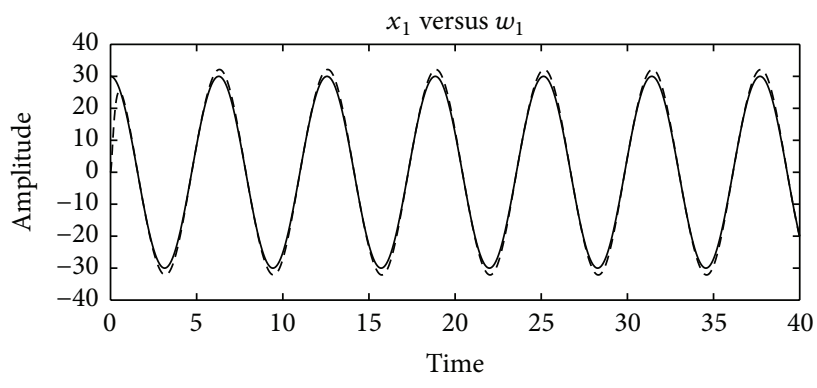

$--x_{1}$

$-w_{1}$

(a)

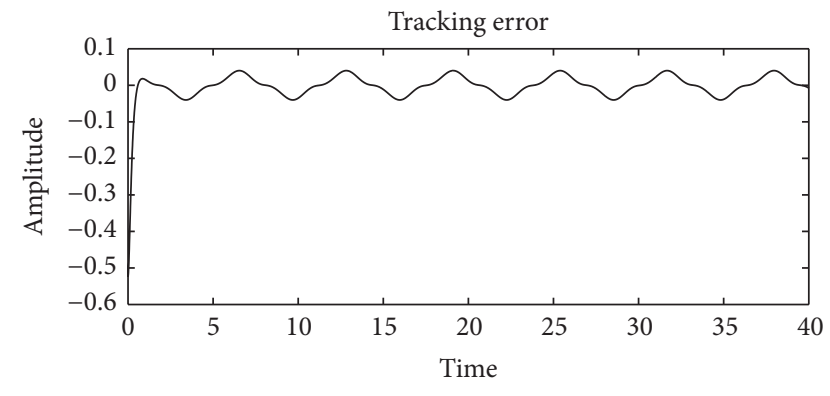

(b)

FIGURE 4: (a) Output reference and (b) tracking error. When the fuzzy controller is designed on local regulators and the adequate membership functions for the fuzzy regulator are computed. 


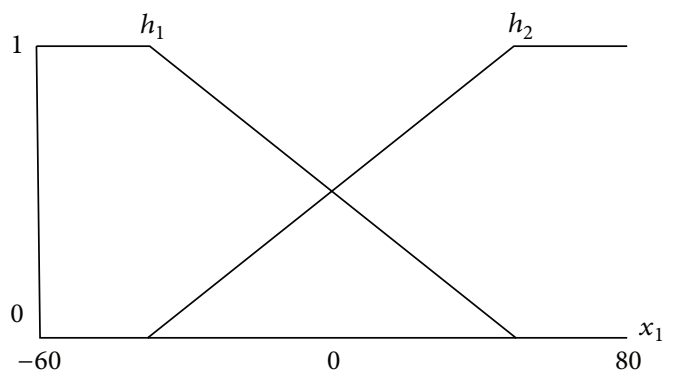

FIGURE 5: Membership functions of two-rule model.

and an exosystem defined by

$$
\begin{gathered}
S=\left[\begin{array}{cccc}
0 & 0 & 1 & 0 \\
0 & 0 & 0 & 1 \\
-4 & 0 & 0 & 0 \\
0 & -16 & 0 & 0
\end{array}\right], \\
Q=\left[\begin{array}{llll}
1 & 0 & 0 & 0 \\
0 & 1 & 0 & 0
\end{array}\right] .
\end{gathered}
$$

Membership functions for Rules 1 and 2 are shown in Figure 5. Matrices defining the local regulators can be easily obtained by using (49). For this example they are

$$
\begin{aligned}
& \Gamma_{1}=\left[\begin{array}{cccc}
1.4286 & 0.5714 & 0.2857 & 0.5714 \\
-1.0000 & -9.5000 & -0.5000 & -2.5000
\end{array}\right], \\
& \Gamma_{2}=\left[\begin{array}{cccc}
2.3333 & 1.3333 & 0.6667 & 0.3333 \\
-0.4000 & -4.0000 & -1.2000 & -0.4000
\end{array}\right] .
\end{aligned}
$$

On the other hand, $q_{1} / c_{1}=\pi_{1}=1, q_{2} / c_{2}=\pi_{2}=1$, $q_{1} / c_{1}=\pi_{3}=1, q_{2} / c_{2}=\pi_{4}=1$, and by (46), and matrix $\Pi$ is

$$
\Pi=\left[\begin{array}{llll}
1 & 0 & 0 & 0 \\
0 & 1 & 0 & 0 \\
0 & 0 & 1 & 0 \\
0 & 0 & 0 & 1
\end{array}\right] .
$$

Figures 6 and 7 show the results obtained using linear regulators defined in (61).

Now, by computing the new membership functions for the fuzzy regulator through (54), one gets

$$
\begin{aligned}
& \mu_{1}(w(t)) \\
& =\left[\begin{array}{cc}
\frac{\left(h_{1}(w(t))\right)(-7)}{h_{1}(w(t))(3)+h_{2}(w(t))(3)} & 0 \\
0 & \frac{\left(h_{1}(w(t))\right)(2)}{h_{1}(w(t))(2)+h_{2}(w(t))(5)}
\end{array}\right], \\
& \mu_{2}(w(t)) \\
& =\left[\begin{array}{cc}
\frac{\left(h_{2}(w(t))\right)(-3)}{h_{1}(w(t))(3)+h_{2}(w(t))(3)} & 0 \\
0 & \frac{\left(h_{2}(w(t))\right)(5)}{h_{1}(w(t))(2)+h_{2}(w(t))(5)}
\end{array}\right] .
\end{aligned}
$$

As previously, the fuzzy stabilizer for this example is also constructed by means of the PDC approach developed in [14], resulting in

$$
\begin{aligned}
& K_{1}=\left[\begin{array}{cccc}
1.0286 & 0.5714 & 0.6000 & 0.5714 \\
-1.0000 & -2.2500 & -0.5000 & -3.7500
\end{array}\right], \\
& K_{2}=\left[\begin{array}{cccc}
1.4000 & 1.3333 & 1.4000 & 0.3333 \\
-0.4000 & -1.1000 & -1.2000 & -0.9000
\end{array}\right] .
\end{aligned}
$$

Thus, Figures 8 and 9 show the simulation results when the controller defined by (20) is applied.

Besides, Figure 10 shows the shape of the new membership functions, which are clearly different from the original ones given in Figure 5.

5.3. More General Problems. Consider the T-S fuzzy MIMO system with multiple inputs defined by the following matrices:

$$
\begin{gathered}
A_{1}=\left[\begin{array}{cccccc}
0 & 0 & 1 & 0 & 0 & 0 \\
0 & 0 & 0 & 1 & 0 & 0 \\
0 & 0 & 0 & 0 & 1 & 0 \\
0 & 0 & 0 & 0 & 0 & 1 \\
-6 & 4 & 2 & 4 & 5 & 3 \\
2 & 3 & -1 & 5 & -2 & 4
\end{array}\right], \\
B_{1}=\left[\begin{array}{ccc}
0 & 0 \\
0 & 0 \\
0 & 0 \\
0 & 0 \\
5 & 0 \\
0 & 2
\end{array}\right], \\
A_{2}=\left[\begin{array}{cccccc}
0 & 0 & 1 & 0 & 0 & 0 \\
0 & 0 & 0 & 1 & 0 & 0 \\
0 & 0 & 0 & 0 & 1 & 0 \\
0 & 0 & 0 & 0 & 0 & 1 \\
-6 & 1 & 2 & -5 & 4 & 6 \\
0 & 2 & -7 & 4 & 5 & 2
\end{array}\right],
\end{gathered}
$$

$$
\begin{gathered}
B_{2}=\left[\begin{array}{ll}
0 & 0 \\
0 & 0 \\
0 & 0 \\
0 & 0 \\
7 & 0 \\
0 & 8
\end{array}\right], \\
C_{1}=C_{2}=\left[\begin{array}{llllll}
0 & 1 & 1 & 0 & 0 & 0 \\
1 & 0 & 1 & 0 & 0 & 0
\end{array}\right] .
\end{gathered}
$$




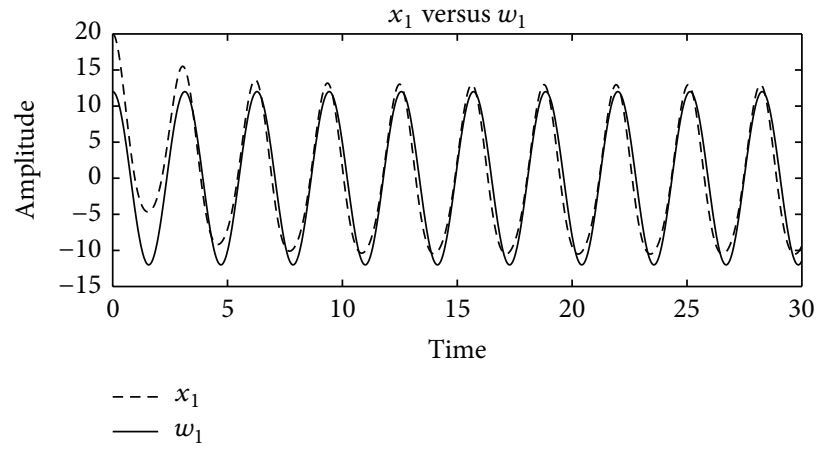

(a)

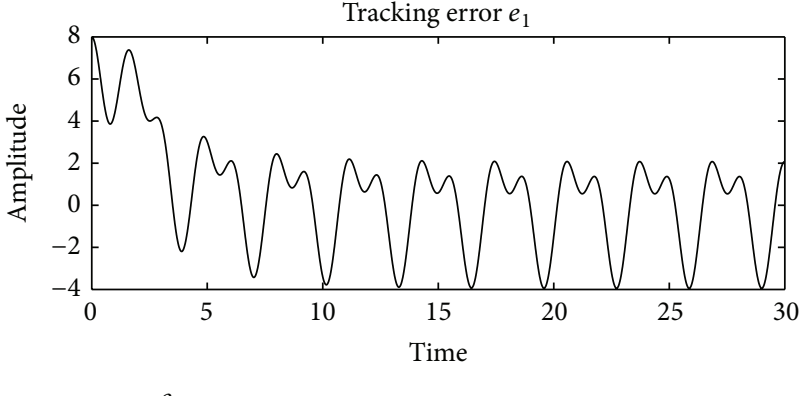

(b)

Figure 6: (a) $x_{1}$ versus $w_{1}$ and (b) tracking error $e_{1}$. When the fuzzy controller is designed on the basis of linear regulators and the fuzzy memberships of the plant are considered in the construction the fuzzy regulator.

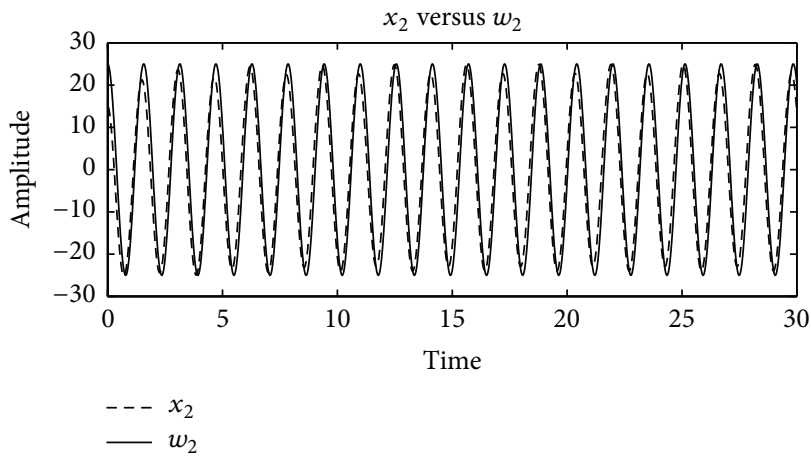

(a)

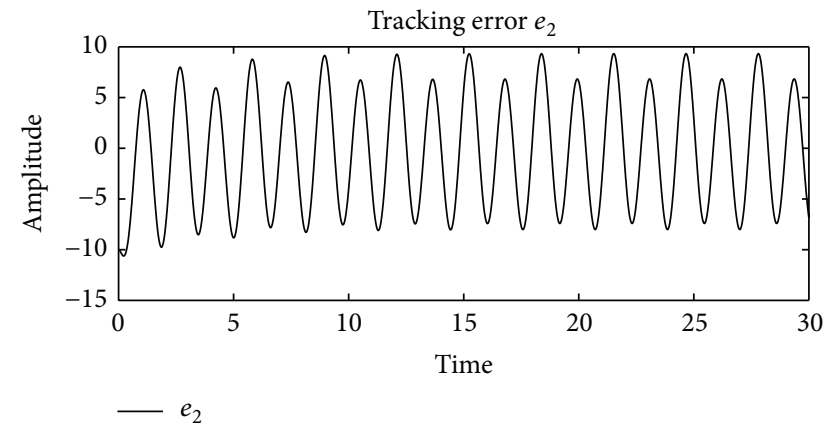

(b)

FIGURE 7: (a) $x_{2}$ versus $w_{2}$ and (b) tracking error $e_{2}$. When the fuzzy controller is designed on the basis of lineal regulators and the fuzzy memberships of the plant are considered in the construction the fuzzy regulator.

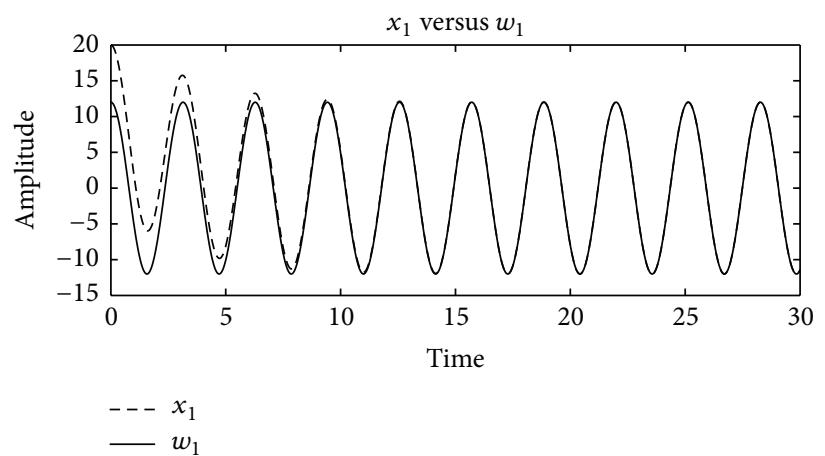

(a)

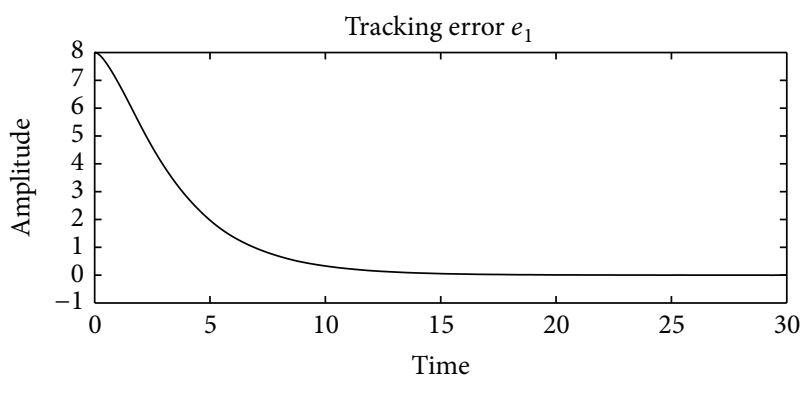

(b)

FIGURE 8: (a) $x_{1}$ versus $w_{1}$ and (b) tracking error $e_{1}$. When the fuzzy controller is designed on the basis of local regulators and the adequate membership functions for the fuzzy regulator are computed.

The reference signals will be generated by an exosystem defined by

$$
S=\left[\begin{array}{cc}
0 & 1 \\
-1 & 0
\end{array}\right], \quad Q=\left[\begin{array}{ll}
1 & 0 \\
0 & 1
\end{array}\right]
$$

Membership functions for Rules 1 and 2 are $h_{1}(x(t))=$ $\left(80-x_{1}(t)\right) / 140$ and $h_{2}(x(t))=1-h_{1}(t)$. Notice that for this case, $n \neq s$ and $C_{i} \neq Q$. This implies that $\Pi_{i}$ and $\Gamma_{i}$ must be obtained by solving Francis Equations for each subsystem. However, due to form of the fuzzy plant it is possible to verify that $\Pi_{1}=\Pi_{2}$. 


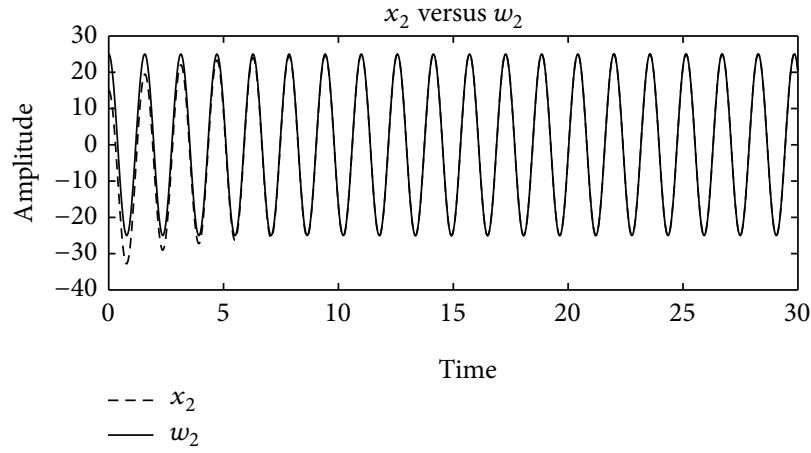

(a)

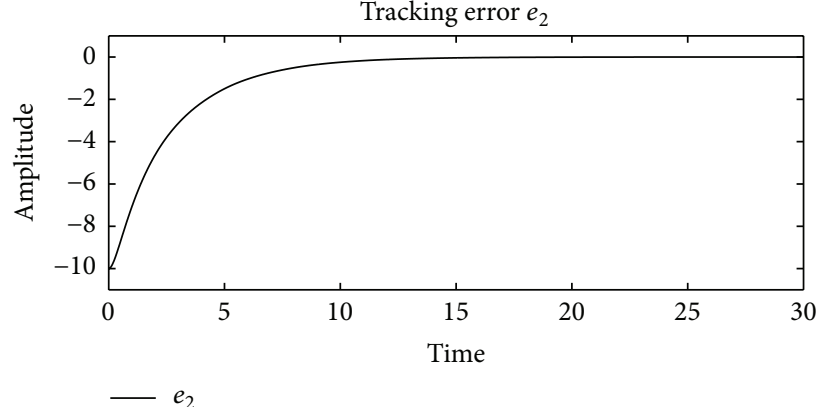

(b)

Figure 9: (a) $x_{2}$ versus $w_{2}$ and (b) tracking error $e_{2}$. When the fuzzy controller is designed on the basis of local regulators and the adequate membership functions for the fuzzy regulator are computed.

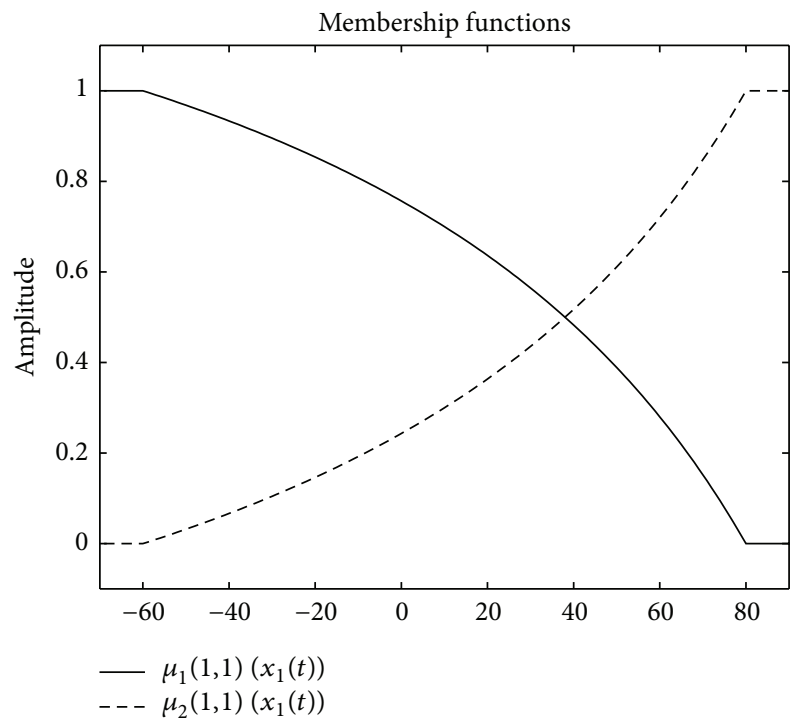

(a)

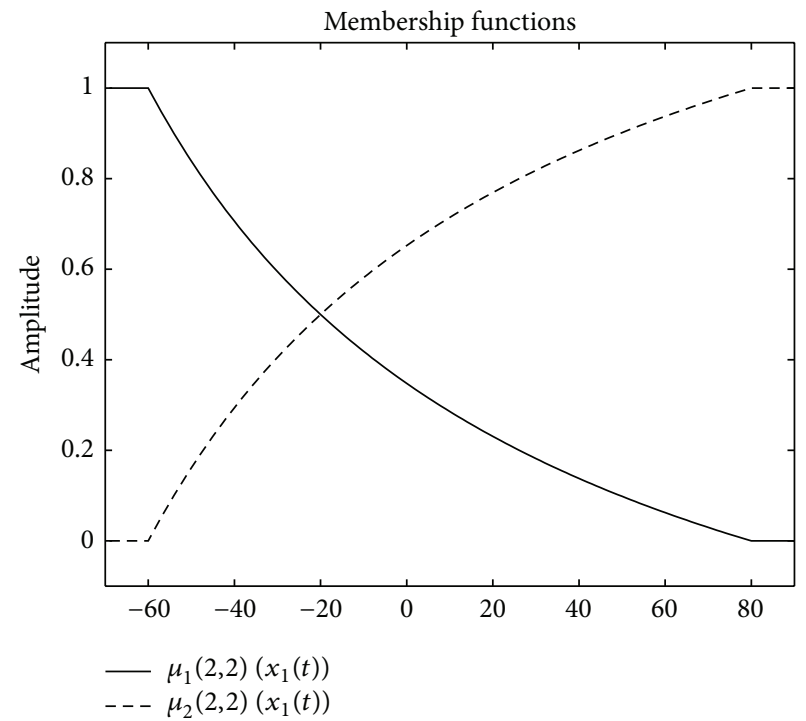

(b)

Figure 10: (a) Membership functions corresponding to position $(1,1)$ of $\mu_{1}(w(t))$ and $\mu_{2}(w(t))$. (b) Membership functions corresponding to position $(2,2) \mu_{1}(w(t))$ and $\mu_{2}(w(t))$.

So, by applying (9) for each local subsystem with $i=1,2$, it results in

$$
\begin{gathered}
\Pi_{1}=\Pi_{2}=\left[\begin{array}{cc}
0.5000 & 0.5000 \\
1.5000 & -0.5000 \\
-0.5000 & 0.5000 \\
0.5000 & 1.5000 \\
-0.5000 & -0.5000 \\
-1.5000 & 0.5000
\end{array}\right], \\
\Gamma_{1}=\left[\begin{array}{cc}
0.7000 & -0.3000 \\
-2.0000 & -5.5000
\end{array}\right], \\
\Gamma_{2}=\left[\begin{array}{cc}
2.3571 & 1.2143 \\
-0.4375 & -0.1875
\end{array}\right] .
\end{gathered}
$$

Again, the fuzzy stabilizer for this system is constructed by means of the PDC approach developed in [14], yielding

$$
\begin{aligned}
& K_{1}=\left[\begin{array}{cccccc}
-1.2229 & 0.0609 & -4.0284 & 0.1291 & -2.5666 & -0.4061 \\
1.6919 & -8.3997 & 3.2934 & -12.7153 & 1.5732 & -6.1836
\end{array}\right], \\
& K_{2}=\left[\begin{array}{llllll}
-0.8735 & 0.4721 & -2.8775 & 1.3780 & -1.6904 & -0.7187 \\
0.6730 & -1.9749 & 1.5734 & -3.0538 & -0.4817 & -1.2959
\end{array}\right] .
\end{aligned}
$$

The behavior obtained by applying controller (61) is depicted in Figures 11 and 12. Notice the exact output regulation is not achieved. 


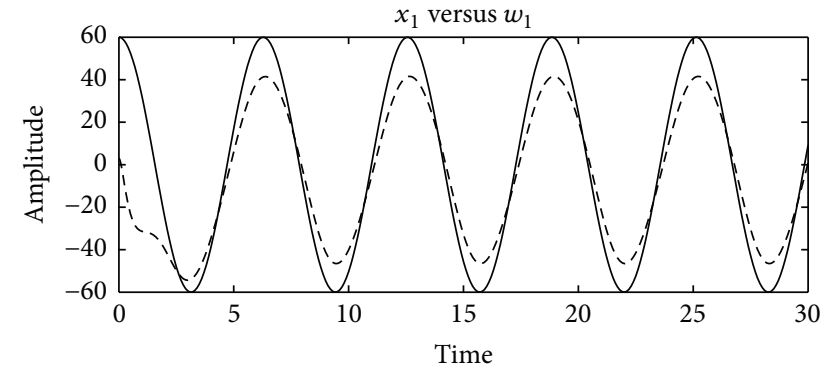

$--x_{1}$

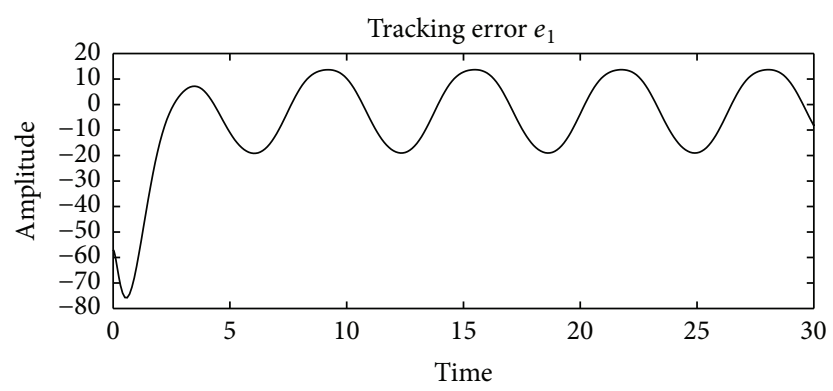

$-e_{1}$

(a)

(b)

Figure 11: (a) $x_{1}$ versus $w_{1}$ and (b) tracking error $e_{1}$. When the fuzzy controller is designed on the basis of linear regulators and the fuzzy memberships of the plant are considered in the construction the fuzzy regulator.

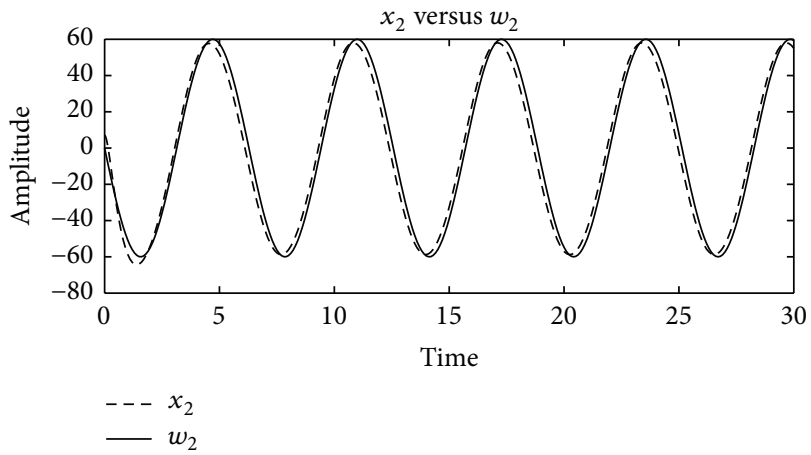

(a)

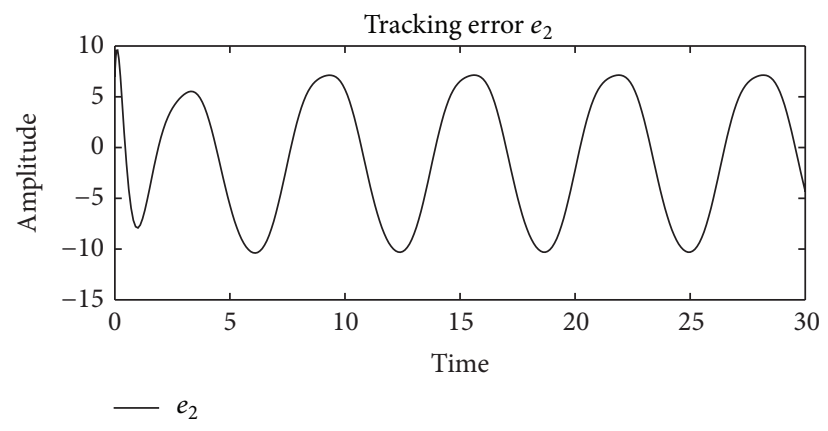

(b)

Figure 12: (a) $x_{2}$ versus $w_{2}$ and (b) tracking error $e_{2}$. When the fuzzy controller is designed on the basis of linear regulators and the fuzzy memberships of the plant are considered in the construction the fuzzy regulator.

Now, the adequate membership functions are computed from (54), resulting in

$\mu_{1}(w(t))$

$=\left[\begin{array}{cc}\frac{\left(h_{1}(w(t))\right)(5)}{h_{1}(w(t))(5)+h_{2}(w(t))(7)} & 0 \\ 0 & \frac{\left(h_{1}(w(t))\right)(2)}{h_{1}(w(t))(2)+h_{2}(w(t))(8)}\end{array}\right]$,

$\mu_{2}(w(t))$

$$
=\left[\begin{array}{cc}
\frac{\left(h_{2}(w(t))\right)(7)}{h_{1}(w(t))(5)+h_{2}(w(t))(7)} & 0 \\
0 & \frac{\left(h_{2}(w(t))\right)(8)}{h_{1}(w(t))(2)+h_{2}(w(t))(8)}
\end{array}\right] .
$$

When $h_{i}(w(t))$ is replaced by its corresponding expressions, one gets

$$
\begin{aligned}
& \mu_{1}(w(t))=\left[\begin{array}{cc}
\frac{1225}{w_{1}(t)+140}-\frac{5}{2} & 0 \\
0 & -\frac{w_{1}(t)-80}{3 w_{1}(t)+320}
\end{array}\right], \\
& \mu_{2}(w(t))=\left[\begin{array}{cc}
\frac{7}{2}-\frac{1225}{w_{1}(t)+410} & 0 \\
0 & \frac{4 w_{1}(t)+240}{3 w_{1}(t)+320}
\end{array}\right] .
\end{aligned}
$$

The results of applying control law (20) on the fuzzy plant are given in Figures 13 and 14.

The "computed" membership functions for the fuzzy regulator are given in Figure 15. Again, it can be readily observed that these new membership functions are different from the original ones.

Notice that, although this example does not fulfill the conditions of Theorem 5 , the membership functions obtained from (39) allow the exact tracking for the fuzzy problem. This 


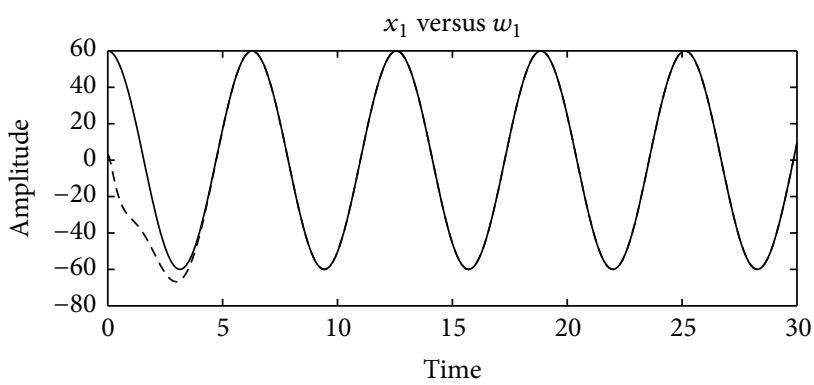

$--x_{1}$

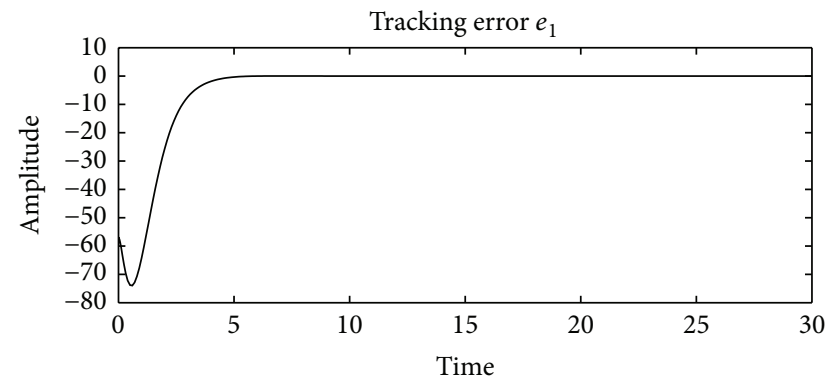

$e_{1}$

(a)

(b)

FIGURE 13: (a) $x_{1}$ versus $w_{1}$ and (b) tracking error $e_{1}$. When the fuzzy controller is designed on the basis of local regulators and the adequate membership functions for the fuzzy regulator are computed.

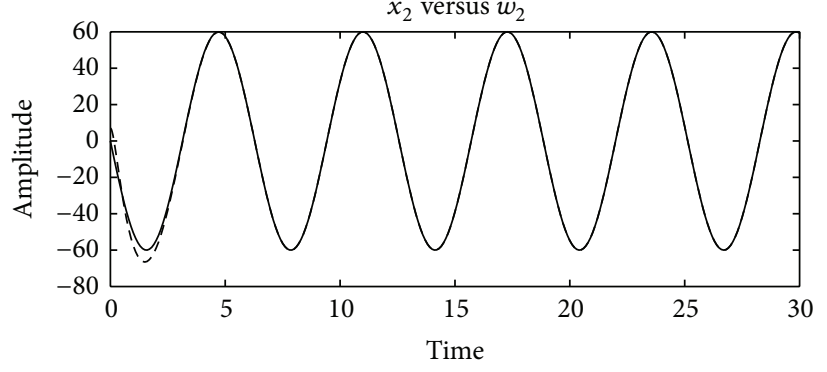

$--x_{2}$

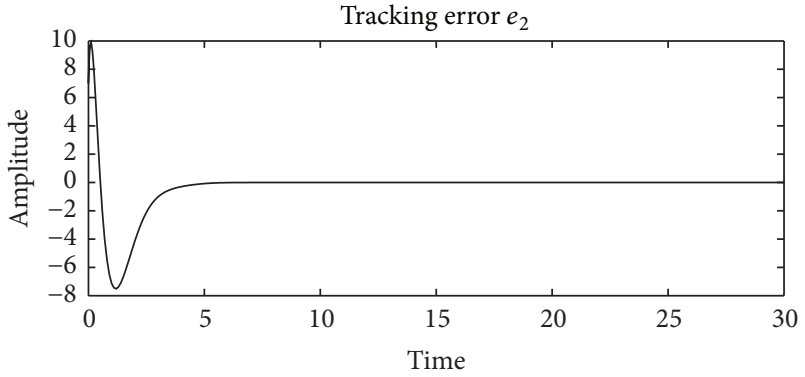

$-e_{2}$

(a)

(b)

FIGURE 14: (a) $x_{2}$ versus $w_{2}$ and (b) tracking error $e_{2}$. When the fuzzy controller is designed on the basis of local regulators and the adequate membership functions for the fuzzy regulator are computed.

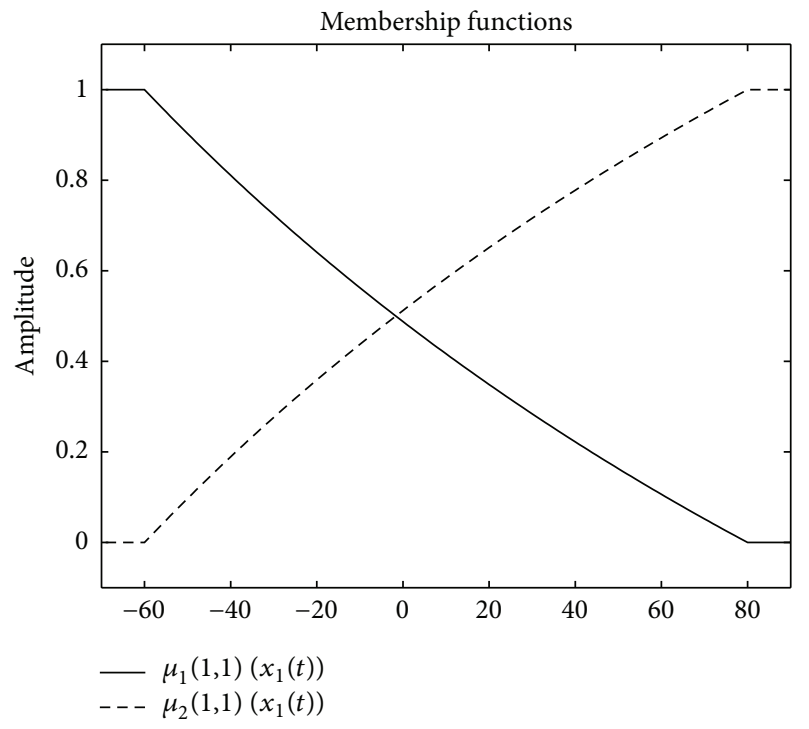

(a)

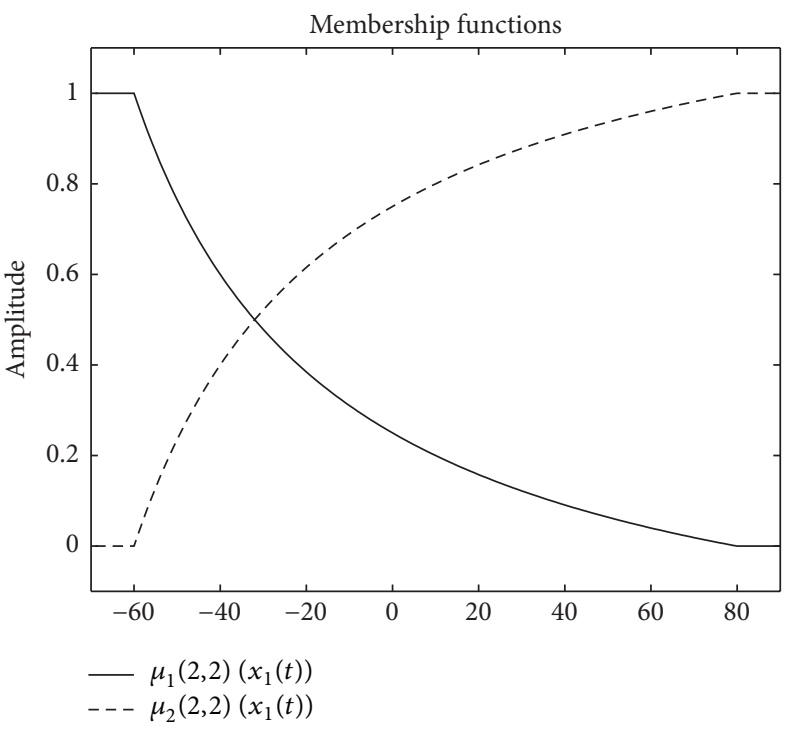

(b)

FIGURE 15: (a) Membership functions corresponding to position $(1,1)$ of $\mu_{1}(w(t))$ and $\mu_{2}(w(t))$. (b) Membership functions corresponding to position $(2,2) \mu_{1}(w(t)), \mu_{2}(w(t))$. 
suggests that the approach presented in this work may be applied on a bigger class of T-S models; however, this study is not completed yet.

\section{Conclusions}

A fuzzy regulator for continuous-time systems, based on the combination of linear regulators adjusted by different membership functions, has been presented. The main advantage is that analytical expressions for the membership functions, which allow the proper combination of the local regulators in order to guarantee the exact regulation, can be easily applied. As a consequence, for a class of T-S fuzzy models, the presented result allows the exact output regulation on the basis of local regulators. Besides, this class of T-S fuzzy models can be commonly found in mechanics, electromechanics, electronics, robotics, and so forth.

Some numerical examples are used to illustrate the applicability of the proposed approach, even in cases where the dimensions of the plant and the exosystem are different between each other. This suggests that the approach presented in this work may be applied on a bigger class of T-S models.

Finally, the method proposed in this work avoids the disadvantage of constructing an exact fuzzy regulator based on overall T-S fuzzy system whose expression may result very large. Instead, the given approach offers a simple way to design the complete regulator based on local regulators but with membership functions which are not necessarily of the same shape as those included in the fuzzy plant.

\section{Conflict of Interests}

The authors declare that there is no conflict of interests regarding the publication of this paper.

\section{Acknowledgments}

This work was partially supported by CONACYT (Consejo Nacional de Ciencia y Tecnología) through scholarship SNI (Sistema Nacional de Investigadores) and by IPN (Instituto Politécnico Nacional) through research Projects 20140659 and 20150487 and scholarships EDI (Estímulo al Desempeño de los Investigadores), COFAA (Comisión de Operación y Fomento de Actividades Académicas), and BEIFI (Beca de Estímulo Institucional de Formación de Investigadores).

\section{References}

[1] B. A. Francis, "The linear multivariable regulator problem," SIAM Journal on Control and Optimization, vol. 15, no. 3, pp. 486-505, 1977.

[2] B. A. Francis and W. M. Wonham, "The internal model principle of control theory," Automatica, vol. 12, no. 5, pp. 457-465, 1976.

[3] A. Isidori and C. I. Byrnes, "Output regulation of nonlinear systems," IEEE Transactions on Automatic Control, vol. 35, no. 2, pp. 131-140, 1990.

[4] B. Castillo-Toledo, S. Di Gennaro, and F. Jurado, "Trajectory tracking for a quadrotor via fuzzy regulation," in Proceedings of the World Automation Congress (WAC '12), pp. 1-6, June 2012.
[5] S. Chen, "Output regulation of nonlinear singularly perturbed systems based on T-S fuzzy model," Journal of Control Theory and Applications, vol. 3, no. 4, pp. 399-403, 2005.

[6] R. Tapia-Herrera, J. A. Meda-Campaña, S. Alc\&apos; antaraMontes, T. Hern\&apos; andez-Cort\&apos; es, and L. SalgadoConrado, "Tuning of a TS fuzzy output regulator using the steepest descent approach and ANFIS," Mathematical Problems in Engineering, vol. 2013, Article ID 873430, 14 pages, 2013.

[7] K.-Y. Lian and J.-J. Liou, "Output tracking control for fuzzy systems via output feedback design," IEEE Transactions on Fuzzy Systems, vol. 14, no. 5, pp. 628-639, 2006.

[8] X.-J. Ma and Z.-Q. Sun, "Output tracking and regulation of nonlinear system based on takagi-sugeno fuzzy model," IEEE Transactions on Systems, Man, and Cybernetics, Part B: Cybernetics, vol. 30, no. 1, pp. 47-59, 2000.

[9] H. J. Lee, J. B. Park, and Y. H. Joo, "Comments on 'output tracking and regulation of nonlinear system based on TakagiSugeno fuzzy model'," IEEE Transactions on Systems, Man, and Cybernetics, Part B: Cybernetics, vol. 33, no. 3, pp. 521-523, 2003.

[10] J. A. Meda-Campaña, B. Castillo-Toledo, and G. Chen, "Synchronization of chaotic systems from a fuzzy regulation approach," Fuzzy Sets and Systems, vol. 160, no. 19, pp. 2860$2875,2009$.

[11] J. A. Meda-Campaña, J. C. G\&apos; omez-Mancilla, and B. Castillo-Toledo, "Exact output regulation for nonlinear systems described by Takagi-Sugeno fuzzy models," IEEE Transactions on Fuzzy Systems, vol. 20, no. 2, pp. 235-247, 2012.

[12] C. Byrnes, F. Priscoli, and A. Isidori, Output Regulation of Uncertain Nonlinear Systems, Birkhäuser, 1997.

[13] A. Isidori, Nonlinear Control Systems, Communications and Control Engineering Series, Springer, Berlin, Germany, 3rd edition, 1995.

[14] K. Tanaka and H. O. Wang, Fuzzy Control Systems Design and Analysis. A Linear Matrix Inequality Approach, John Wiley \&amp; Sons, New York, NY, USA, 2001.

[15] L.-X. Wang, A Course in Fuzzy Systems and Control, Prentice Hall, Upper Saddle River, NJ, USA, 1997.

[16] I. Abdelmalek, N. Gol\&apos; ea, and M. L. Hadjili, "A new fuzzy Lyapunov approach to non-quadratic stabilization of Takagi-Sugeno fuzzy models," International Journal of Applied Mathematics and Computer Science, vol. 17, no. 1, pp. 39-51, 2007.

[17] J. A. Meda-Campaña and B. Castillo-Toledo, "On the output regulation for TS fuzzy models using sliding modes," in Proceedings of the American Control Conference (ACC '05), pp. 40624067, Portland, Ore, USA, June 2005.

[18] B. Castillo-Toledo and J. A. Meda-Campaña, "The fuzzy discrete-time robust regulation problem: a LMI approach," in Proceedings of the 41st IEEE Conference on Decision and Control, vol. 2, pp. 2159-2164, IEEE, Las Vegas, Nev, USA, December 2002.

[19] B. Castillo-Toledo, J. A. Meda-Campaña, and A. Titli, "A fuzzy output regulator for Takagi-Sugeno fuzzy models," in Proceedings of the IEEE International Symposium on Intelligent Control, pp. 310-315, Houston, Tex, USA, October 2003.

[20] B.-C. Ding, H.-X. Sun, and Y.-E. Qiao, "Stability analysis of T-S fuzzy control systems based on parameter-dependent Lyapunov function," Acta Automatica Sinica, vol. 31, no. 4, pp. 651-654, 2005.

[21] M. Narimani, H. K. Lam, R. Dilmaghani, and C. Wolfe, "LMIbased stability analysis of fuzzy-model-based control systems using approximated polynomial membership functions," IEEE 
Transactions on Systems, Man, and Cybernetics, Part B: Cybernetics, vol. 41, no. 3, pp. 713-724, 2011.

[22] H. K. Lam, "Polynomial fuzzy-model-based control systems: stability analysis via piecewise-linear membership functions," IEEE Transactions on Fuzzy Systems, vol. 19, no. 3, pp. 588-593, 2011.

[23] K. Tanaka, T. Hori, and H. O. Wang, "A fuzzy Lyapunov approach to fuzzy control system design," in Proceedings of the American Control Conference, vol. 6, pp. 4790-4795, Arlington, Va, USA, June 2001.

[24] K. Tanaka, T. Hori, and H. O. Wang, "A multiple Lyapunov function approach to stabilization of fuzzy control systems," IEEE Transactions on Fuzzy Systems, vol. 11, no. 4, pp. 582-589, 2003.

[25] S. Zhou, G. Feng, J. Lam, and S. Xu, "Robust $H_{\infty}$ control for discrete-time fuzzy systems via basis-dependent Lyapunov functions," Information Sciences, vol. 174, no. 3-4, pp. 174-197, 2005. 


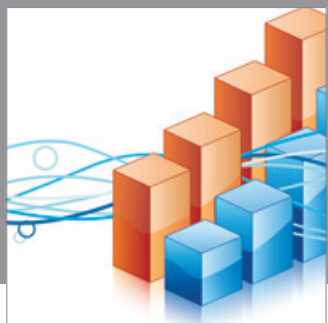

Advances in

Operations Research

mansans

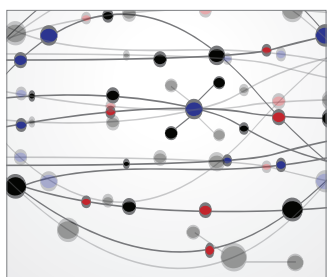

The Scientific World Journal
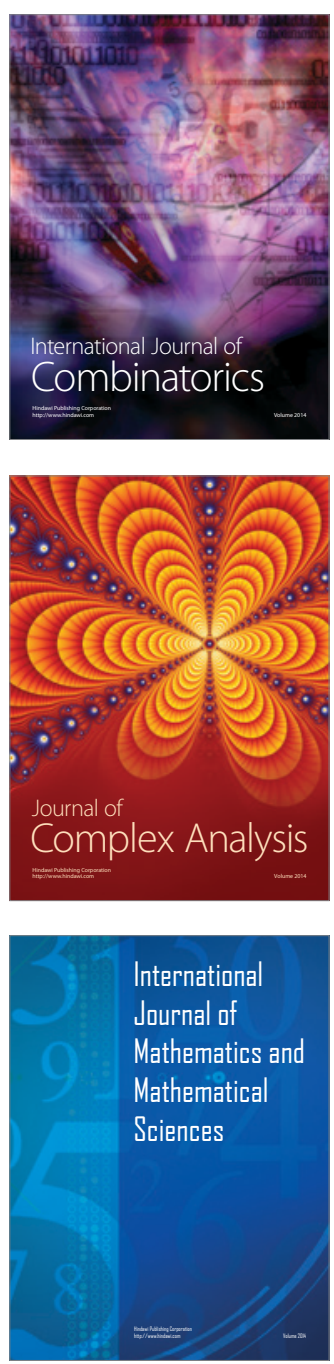
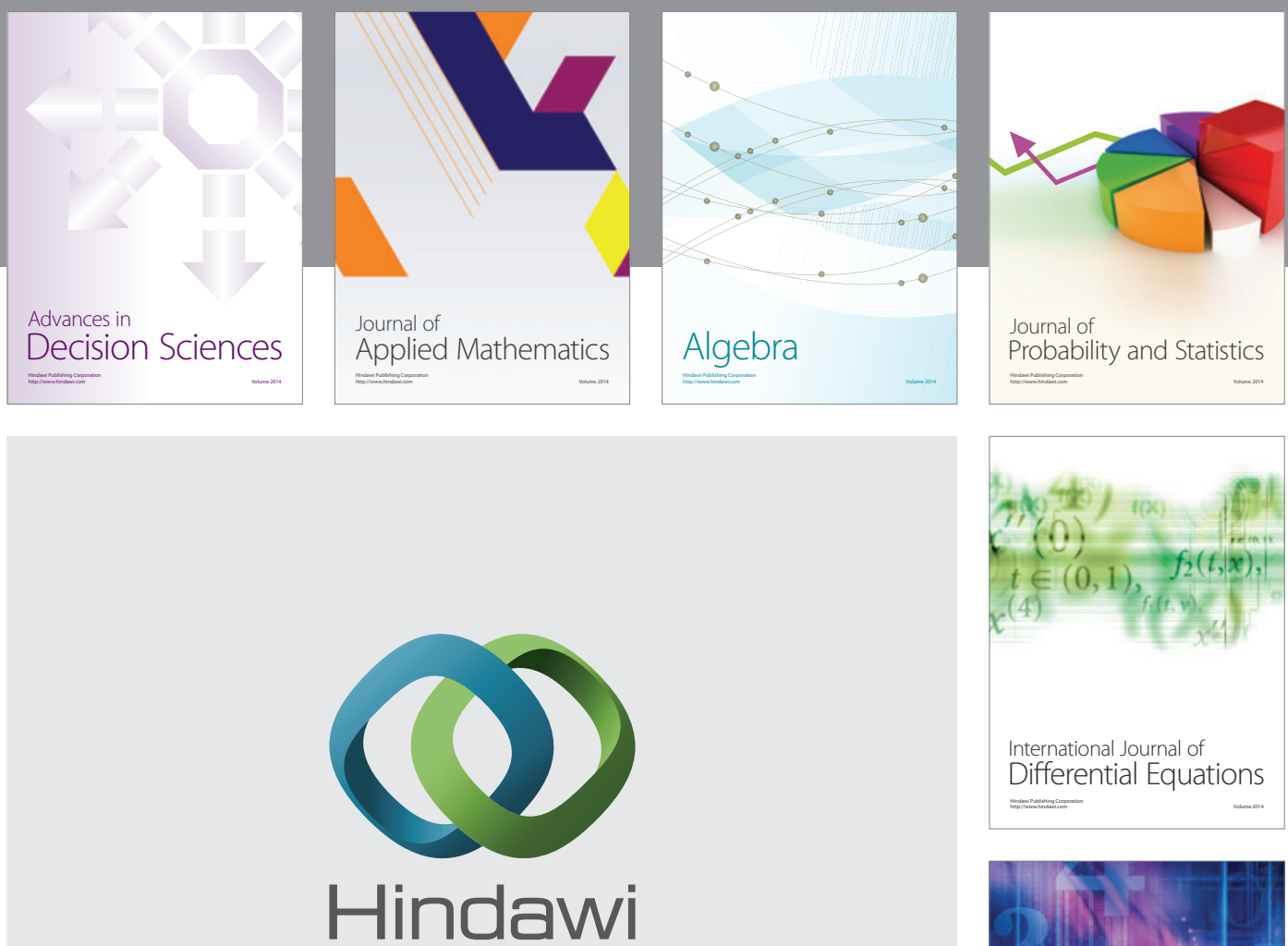

Submit your manuscripts at http://www.hindawi.com
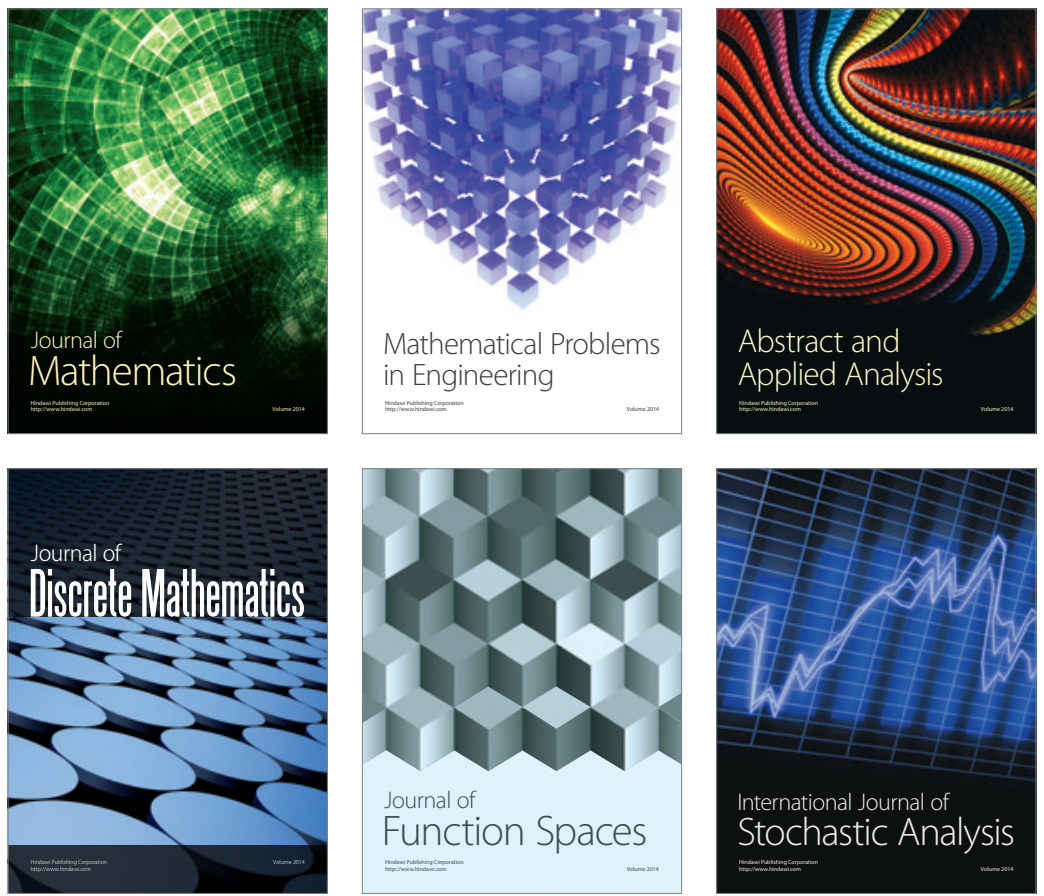

Journal of

Function Spaces

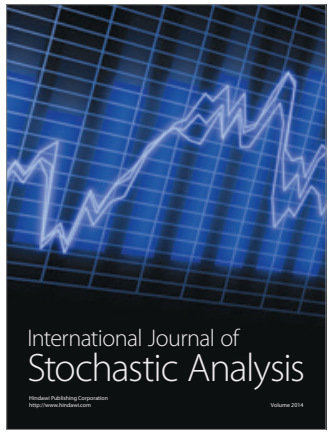

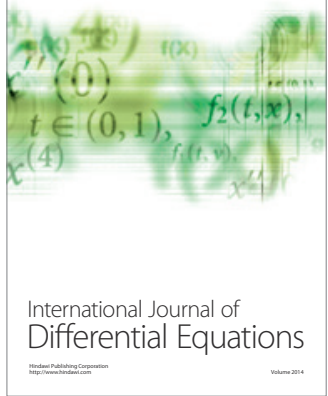
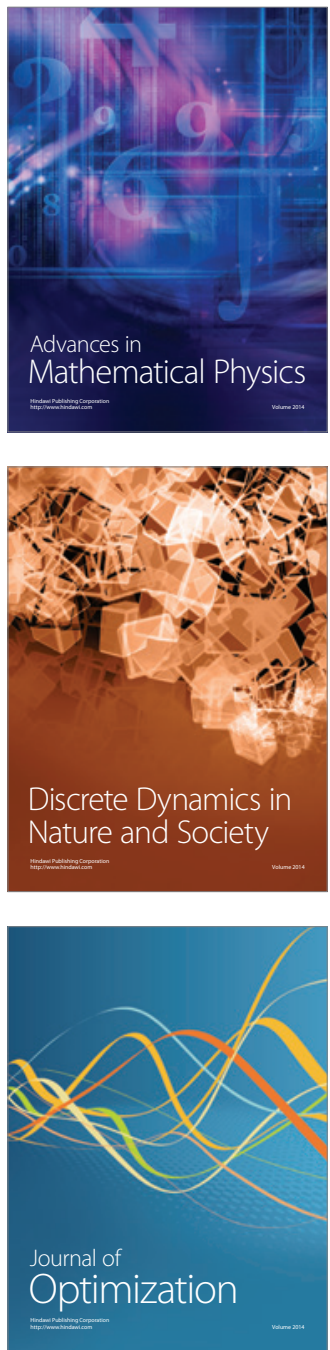